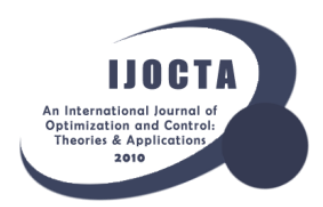

\title{
Dynamics of malaria-dengue fever and its optimal control
}

\author{
Nita H. Shah*, Ankush H. Suthar, Ekta N. Jayswal \\ Department of Mathematics, Gujarat University, Ahmedabad, India-380009 \\ nitahshah@gmail.com,ankush.suthar1070@gmail.com,jayswal.ekta1993@gmail.com
}

\section{ARTICLE INFO}

Article history:

Received: 3 June 2019

Accepted: 20 December 2019

Available Online: 5 June 2020

Keywords:

Malaria-dengue

Dynamical system

Basic reproduction number

Stability

Optimal control

Numerical Simulation

AMS Classification 2010:

$37 N x x, 37 M 05,93 D 05$

\begin{abstract}
The mosquito-borne infectious diseases like malaria and dengue are putative as important tropical infections and cause high morbidity and mortality around the world. In some cases, simultaneous coexistence of both the infections in one individual is seen which is very hard to distinguish as both diseases have almost similar symptoms. In this proposed article, dynamical system of non-linear differential equations is constructed with the help of mathematical modeling, which describe dynamics of the spread of these infectious diseases separately and concurrently. Basic reproduction number is evaluated to understand dynamical behaviour of the model. Local and global stability criteria have been deliberated rigorously. Control parameters are used to perceive effect of medication on these prevalent tropical diseases. Numerical simulations are used to observe effect of control parameters graphically.
\end{abstract}

\section{Introduction}

The present era witneses the globalization of infectious diseases that occurs frequently by an unprecedented level. In this "globalized" environment of interdependent trade, travel, migration, and international economic markets, many factors now play an important role in the emergence and spread of infectious disease, which necessitates a coordinated, global response [17]. Mosquitoes are one of the deadliest insects in the world, with their ability to carry and spread disease to humans causes millions of deaths every year. Mosquito-borne infectious disease is accepted as one of the important tropical infections and is the focused topic in tropical medicine [23]. There are several tropical mosquito borne infections. Malaria and dengue are the two common mosquito infections that are easily spread and cause high morbidity and mortality for many patients around the world. Malaria is caused by Plasmodium parasites, which spreads through the bites of infected female Anopheles mosquitoes, called 'malaria vectors' [18]. Dengue is single positive-stranded RNA virus of the family Flaviviridae which is ingested by female mosquitoes (Aedes mosquito) during feeding [22]. The virus then infects the other mosquito and humans over its incubation period. Due to tremendous progress in malaria and dengue infection, the disease burden remains high mostly in subtropical and tropical areas [21].

Presence of infection in the body results in weakness in immune system, it increases the probability that individual gets infected by another infections. Hence there is a possibility that both malaria and dengue infection can be present in the individual at the same time (e.g., [4], [6], [8], [13], [21], [24], [30] or [13]). This scenario is called concurrent malaria-dengue infection. This overlapping of two different infections can result in more severe situations where both diagnosis and treatment of a patient may become difficult [10]. Initially, two cases of concurrent malaria and dengue infection were identified in July, 2005 and November, 2006 [4]. Malaria and dengue fever represent 2 major public health concerns in South America, whose $92 \%$ of area is covered by Amazon rain forest. According to the report in a French territory in South America, 0.99\% from overall febrile patients are infected by malaria and dengue concurrently [4]. Malaria vectors and dengue vectors are habited in the forest [20] and in the city [7] respectively. Hence, overlapping of the habitat cannot be easily available and therefore concurrent malaria dengue infection cases are less in number.

Mathematical models relevant to the concurrent infections helps the researchers, biologists and public 
health personnel to adopt improved and most effective strategies to control the diseases. Aldila D. and Agustin M. R. developed a nine-dimensional mathematical model to understand the spread of dengue and chikungunya in a closed population [1]. Isea R. \& Lonngren K. E. presented two preliminary models that consist of the individual transmission dynamics of dengue, Chikungunya or Zika, and any possible coinfection between two diseases in the same population [12]. Sharomi et. al. developed a deterministic model which incorporates many of the essential biological and epidemiological features of HIV and tuberculosis and the synergistic interaction between them [25]. Silva C. J. \& Torres D. F. proposed a population and introduced optimal treatment strategies for co-infection transmission dynamics of TB and HIV [26].

Some cases are reported where patients have symptoms of malaria and dengue both at the same time. In such situations, higher mortality rate is observed. On the basis of this observation, a mathematical model is constructed in the present work. Also two optimal controls are applied in the model in such a way that it helps to analyse malaria-dengue concurrent case and effect of recovery rate on the disease transmission. The paper is organized as follows. The malaria-dengue model construction will be discussed in section 2 . Section 3 focuses on formulating basic reproduction number for concurrent malaria-dengue infection, moreover the equilibrium points of the given model are calculated. Local and global stability of all four equilibrium points are proved in section 4. Optimal control theory is introduced and applied to the model in section 5. The model is analysed numerically and graphically in the next section which provides better explanation of the analytic results.

\section{Mathematical modeling}

The environmental stress also damages the immune system and makes the individual weak to resist various kinds of infections. Motivated from this concurrent disease problem, we have proposed a compartmental model to analyze the spread of malaria and dengue infections individually and concurrently. The model subdivides the human population $(N)$ into four mutually-exclusive compartments, namely susceptible individuals $(S)$, malaria infected individuals $(M)$, dengue infected individuals $(D)$ and corresponding to two infectious agent class of recovered individuals is $(R)$. Total recruitment rate in class of susceptible at time $t$ is $B$. Susceptible individuals are infected by malaria infection with transmission rate $\alpha_{1}$. The disease transmission from the class of susceptible individuals to the class of dengue infected individuals is taken as a saturated form with disease transmission rate $\alpha_{2}$ and $\alpha_{3}$ be the reciprocal of the half saturation constant. Therefore, from compartment $S$ to $D$ the disease transmission form is taken as $\frac{\alpha_{2} S D}{1+\alpha_{3} D}$. The parameter $\alpha_{4}$ represents the rate of the malaria infection giving rise to the dengue infection due to weak immunity. $\alpha_{5}$ and $\alpha_{6}$ are the rates at which the population infected by malaria and dengue are recovered respectively. $\mu$ is assumed as a natural death rate and $\mu_{D}$ be the dengue infection related death rate.

In Figure 1 the schematic diagram of the transmission of disease is shown. Here a concurrent disease case in which individual first get affected by dengue and then by malaria is ignored.

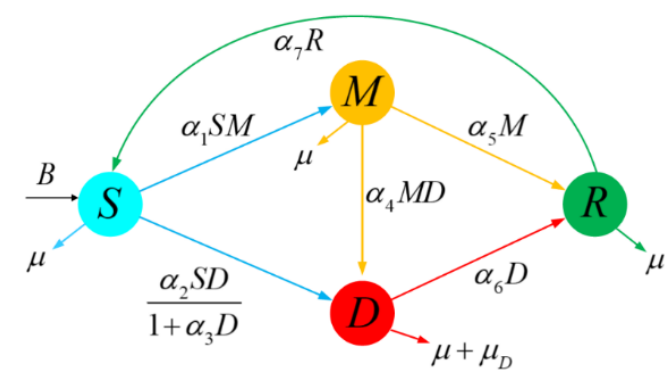

Figure 1. Schematic diagram of malaria-dengue model

On the basis these assumptions and figure 1, we formulate our model as:

$\frac{d S}{d t}=B+\alpha_{7} R-\alpha_{1} S M-\frac{\alpha_{2} S D}{1+\alpha_{3} D}-\mu S$

$\frac{d M}{d t}=\alpha_{1} S M-\alpha_{4} M D-\alpha_{5} M-\mu M$

$\frac{d D}{d t}=\frac{\alpha_{2} S D}{1+\alpha_{3} D}+\alpha_{4} M D-\alpha_{6} D-\left(\mu+\mu_{D}\right) D$

$\frac{d R}{d t}=\alpha_{6} D+\alpha_{5} M-\alpha_{7} R-\mu R$

The initial conditions of the system (1) are $S(0)>0$, $M(0)>0, D(0)>0, R(0)>0$.

\section{Basic reproduction number $\left(R_{0}\right)$ and equilibrium}

EnvironmentsNote that $S+M+D+R=N$ and all the compartments are taken positive. Summing all the equations of the system (1) gives,

$\frac{d}{d t}(S+M+D+R)=B-\mu(S+M+D+R)-\mu_{D} D \geq 0$

Hence, $\lim _{t \rightarrow \infty} \sup (S+M+D+R) \leq \frac{B}{\mu}$

Therefore, the feasible region for system (1) is:

$\Lambda=\left\{\begin{array}{r}(S+M+D+R) /(S+M+D+R) \leq \frac{B}{\mu} ; \\ S \geq 0, M \geq 0, D \geq 0, R \geq 0\end{array}\right\}$ 
Clearly the point $E_{0}=\left(S_{0}, 0,0,0\right)$, where $S_{0}=\frac{B}{\mu}$ is an equilibrium point of the system (1), which is called a disease free equilibrium point. The model has three more equilibrium points as follows,

I. Dengue free equilibrium point $E_{1}=\left(S_{1}, M_{1}, 0, R_{1}\right)$ where, $S_{1}=\frac{\alpha_{5}+\mu}{\alpha_{1}}, M_{1}=\frac{\left(\alpha_{7}+\mu\right) k_{1}}{\alpha_{1} \mu}$, $R_{1}=\frac{\alpha_{5} k_{1}}{\alpha_{1} \mu}$ and $k_{1}=\frac{\left(B \alpha_{1}-\alpha_{5} \mu-\mu^{2}\right)}{\mu\left(\alpha_{5}+\alpha_{7}+\mu\right)}$

II. Malaria free equilibrium point $E_{2}=\left(S_{2}, 0, M_{2}, R_{2}\right)$ where, $M_{2}=k_{2}\left(\alpha_{7}+\mu\right)$, $R_{2}=\alpha_{6} k_{2}$, where

$S_{2}=\frac{k_{2}\left(\begin{array}{l}\left(\alpha_{7}+\mu\right)\left(\left(\mu_{D}+\alpha_{6}\right)\left(B \alpha_{3}+\mu_{D}+\mu\right)\right. \\ \left.+\mu\left(\mu_{D} \mu+B \alpha_{3}+\mu_{D} \alpha_{6}\right)\right)+\alpha_{6} \mu\left(\alpha_{6}+\mu\right)\end{array}\right)}{\mu\left(\mu_{D}+\mu\right)+\alpha_{6} \mu-B \alpha_{2}}$ and $k_{2}=\frac{\mu\left(\mu_{D}+\mu\right)+\alpha_{6} \mu-B \alpha_{2}}{\left(\begin{array}{l}\left(\alpha_{7}+\mu\right)\left(\mu_{D} \alpha_{3} \mu+\alpha_{3} \mu\left(\alpha_{6}+1\right)+\alpha_{2}\left(\mu_{D}+\mu\right)\right) \\ +\alpha_{2} \alpha_{6} \mu\end{array}\right)}$

III. Endemic equilibrium point

$$
\begin{aligned}
& E^{*}=\left(S^{*}, M^{*}, D^{*}, R^{*}\right), \text { where } \\
& S^{*}=\frac{\alpha_{4} k_{3}+\alpha_{5}+\mu}{\alpha_{1}}, D^{*}=k_{3},
\end{aligned}
$$

$M^{*}=-\frac{\left(\alpha_{7}+\mu\right)\left(\alpha_{1} k_{3}\left(\mu_{D}+\mu\right)+\mu\left(\alpha_{4} k_{3}+\alpha_{5}+\mu\right)-B \alpha_{1}\right)}{\alpha_{1} \mu\left(\alpha_{5}+\alpha_{7}+\mu\right)}$

$R^{*}=-\frac{\left(\begin{array}{l}\alpha_{1} \alpha_{5} k_{3}\left(\mu_{D}+\mu\right)-k_{3} \mu\left(\alpha_{4} \alpha_{5}-\alpha_{1} \alpha_{6}\right) \\ -B \alpha_{1} \alpha_{5}+\alpha_{5} \mu\left(\alpha_{5}+\mu\right)\end{array}\right)}{\alpha_{1} \mu\left(\alpha_{5}+\alpha_{7}+\mu\right)}$,

$k_{3}=$ Root of $\left\{\left(\left(\alpha_{7}+\mu\right)\left(\alpha_{3} \alpha_{4}\left(\mu_{D} \alpha_{1}+\alpha_{1} \mu+\alpha_{4} \mu\right)\right)\right.\right.$

$\left.+\alpha_{1} \alpha_{3} \alpha_{4} \alpha_{6} \mu\right) z^{2}+\left(\left(\alpha_{7}+\mu\right)\left(\left(\alpha_{1} \alpha_{3} \mu+\alpha_{1} \alpha_{4}\right)\left(\mu_{D}+\mu\right)\right.\right.$

$+\alpha_{3} \alpha_{4} \mu\left(\alpha_{5}+\mu\right)+\alpha_{1} \alpha_{3}\left(B \alpha_{4}+\alpha_{6} \mu\right)$

$\left.+\alpha_{4} \mu\left(\alpha_{4}-\alpha_{2}\right)\right)+\alpha_{1} \alpha_{3} \alpha_{5} \mu\left(\alpha_{6}+\mu\right)$

$\left.+\mu_{D} \alpha_{1} \alpha_{3} \alpha_{5} \mu+\alpha_{1} \alpha_{4} \alpha_{6} \mu-\alpha_{2} \alpha_{4} \alpha_{5} \mu\right) z$

$+\left(\alpha_{7}+\mu\right)\left(\alpha_{1} \mu\left(\alpha_{6}+\mu\right)+\alpha_{1}\left(\mu_{D} \mu-B \alpha_{4}\right)\right.$

$\left.+\mu\left(\alpha_{4}-\alpha_{2}\right)\left(\alpha_{5}+\mu\right)\right)+\alpha_{1} \alpha_{5} \mu\left(\alpha_{6}+\mu\right)$

$\left.-\alpha_{2} \alpha_{5} \mu\left(\alpha_{5}+\mu\right)+\mu_{D} \alpha_{1} \alpha_{5} \mu\right\}$

Since the threshold parameter is useful in characterizing the spread of an infectious disease. Here, we use the next generation matrix ([9], [28], [3]) to obtain the expression of basic reproduction number $R_{0}$ for concurrent malaria-dengue infection.

Let $X=(S+M+D+R)$, then system (1) can be written as $X^{\prime}=\mathcal{F}(X)-\mathscr{V}(X)$ such that,

$$
\begin{gathered}
\mathcal{F}=\left[\begin{array}{c}
\alpha_{1} S M \\
\frac{\alpha_{2} S D}{1+\alpha_{3} D}+\alpha_{4} M D \\
0 \\
0
\end{array}\right], \\
\mathscr{V}=\left[\begin{array}{c}
\alpha_{4} M D+\alpha_{5} M+\mu M \\
\alpha_{6} D+\mu D+\mu_{D} D \\
-\alpha_{6} D-\alpha_{5} M+\alpha_{7} R+\mu R \\
-B-\alpha_{7} R+\alpha_{1} S M+\frac{\alpha_{2} S D}{1+\alpha_{3} D}+\mu S
\end{array}\right]
\end{gathered}
$$

Let matrices $\mathcal{F}$ and $\mathcal{V}$ are be the Jacobian of $\mathcal{F}$ and $\vartheta$ respectively around disease free equilibrium point $\left(E_{0}\right)$ :

$$
\mathcal{F}=\left[\begin{array}{cccc}
\frac{\alpha_{1} B}{\mu} & 0 & 0 & 0 \\
0 & \frac{\alpha_{2} B}{\mu} & 0 & 0 \\
0 & 0 & 0 & 0 \\
0 & 0 & 0 & 0
\end{array}\right],
$$

$$
\mathcal{V}=\left[\begin{array}{cccc}
\alpha_{5}+\mu & 0 & 0 & 0 \\
0 & \mu+\mu_{D}+\alpha_{6} & 0 & 0 \\
-\alpha_{5} & -\alpha_{6} & \alpha_{7}+\mu & 0 \\
\frac{\alpha_{1} B}{\mu} & \frac{\alpha_{2} B}{\mu} & -\alpha_{7} & \mu
\end{array}\right]
$$

Here, the matrix $\mathcal{F}$ is related to the rate of increase of new individual in compartment and $\mathcal{V}$ to the rate of the diseases transmission in compartments.

The next generation matrix $K=\left(\mathcal{F} \mathcal{V}^{-1}\right)$ have non negative eigenvalues. The basic reproduction number $R_{0}$ for the model is the spectral radius of $\left.K=(\mathcal{F})^{-1}\right)$ , which is:

$R_{0}=\frac{\alpha_{1} B}{\mu\left(\alpha_{5}+\mu\right)}+\frac{\alpha_{2} B}{\mu\left(\mu_{D}+\alpha_{6}+\mu\right)}$

\section{Stability analysis}

This section includes stability results of all the equilibrium points of the proposed malaria-dengue model.

\subsection{Local stability}

Local stability of all the equilibrium points has been established by following theorems.

Theorem 1. The disease free equilibrium point $E_{0}$ of model is locally asymptotically stable if it satisfy following two conditions.

$$
\text { I. } \frac{\alpha_{1} B}{\mu}>\alpha_{5}+\mu
$$




$$
\text { II. } \frac{\alpha_{2} B}{\mu}>\mu_{D}+\alpha_{6}+\mu
$$

Proof. Jacobian matrix of the model around point $E_{0}$ is:

$$
J\left(E_{0}\right)=\left[\begin{array}{cccc}
-\mu & \frac{-\alpha_{1} B}{\mu} & \frac{-\alpha_{2} B}{\mu} & \alpha_{7} \\
0 & \frac{\alpha_{1} B}{\mu}-\alpha_{5}-\mu & 0 & 0 \\
0 & 0 & \frac{\alpha_{2} B}{\mu}-\mu_{D}-\alpha_{6}-\mu & 0 \\
0 & \alpha_{5} & \alpha_{6} & -\alpha_{7}-\mu
\end{array}\right]
$$

Eigenvalues of the matrix $J\left(E_{0}\right)$ are $\lambda_{1}^{0}=-\mu$,

$$
\lambda_{2}^{0}=\frac{\alpha_{1} B}{\mu}-\alpha_{5}-\mu, \lambda_{3}^{0}=\frac{\alpha_{2} B}{\mu}-\mu_{D}-\alpha_{6}-\mu,
$$

$\lambda_{4}^{0}=-\left(\alpha_{7}+\mu\right)$.

Clearly all the eigenvalues are negative if $\frac{\alpha_{1} B}{\mu}>\alpha_{5}+\mu$ and $\frac{\alpha_{2} B}{\mu}>\mu_{D}+\alpha_{6}+\mu$, hence disease

free equilibrium point is locally asymptotically stable under these conditions.

Theorem 2. The dengue free equilibrium point $E_{1}$ is locally asymptotically stable if it satisfy following two conditions.

$$
\begin{array}{ll}
\text { I. } & B \alpha_{1}>\mu\left(\alpha_{5}+\mu\right) \\
\text { II. } & \alpha_{2}\left(\alpha_{5}+\mu\right)<\alpha_{1}\left(\mu_{D}+\mu\right) \text { and } \alpha_{6} \mu>B \alpha_{5}
\end{array}
$$

Proof. Jacobian matrix of the model around point $E_{1}$ is:

$$
J\left(E_{1}\right)=\left[\begin{array}{cccc}
-n_{1}-\mu & -n_{5}-\mu & -n_{2} & \alpha_{7} \\
n_{1} & 0 & -\frac{\alpha_{4} n_{1}}{\alpha_{1}} & 0 \\
0 & 0 & n_{2}+\frac{\alpha_{4} n_{1}}{\alpha_{1}}-\mu_{D}-\alpha_{6}-\mu & 0 \\
0 & \alpha_{5} & \alpha_{6} & -\left(\alpha_{7}+\mu\right)
\end{array}\right]
$$

Where, $n_{1}=\frac{\left(B \alpha_{1}-\alpha_{5} \mu-\mu^{2}\right)\left(\alpha_{7}+\mu\right)}{\mu\left(\alpha_{5}+\alpha_{7}+\mu\right)}$ and $n_{2}=\frac{\alpha_{2}\left(\alpha_{5}+\mu\right)}{\alpha_{1}}$

Eigenvalues of the Jacobian matrix $J\left(E_{1}\right)$ are:

$$
\begin{aligned}
& \lambda_{1}^{1}=-\mu, \lambda_{2}^{1}=-\frac{1}{2 \mu\left(\alpha_{5}+\alpha_{7}+\mu\right)}\left(\beta_{1}-\sqrt{\beta_{2}}\right), \\
& \lambda_{3}^{1}=- \frac{\left(\beta_{1}+\sqrt{\beta_{2}}\right)}{2 \mu\left(\alpha_{5}+\alpha_{7}+\mu\right)}, \\
&\left(( \alpha _ { 7 } + \mu ) \left(\alpha_{1} \mu\left(\mu_{D}+\mu\right)\left(1+\alpha_{5}\right)-B \alpha_{1} \alpha_{5}\right.\right. \\
& \lambda_{4}^{1}=-\left.+\alpha_{1} \alpha_{6} \mu+\left(\alpha_{5}+\mu\right)\left(\alpha_{4} \mu-\alpha_{2} \mu\left(1+\alpha_{5}\right)\right)\right) \\
& \alpha_{1} \mu\left(\alpha_{5}+\alpha_{7}+\mu\right)
\end{aligned}
$$

Where, $\beta_{1}=\left(\alpha_{7}+\mu\right)\left(B \alpha_{1}+\mu \alpha_{7}\right)$,

$$
\begin{aligned}
& \beta_{2}=\left(\alpha_{7}+\mu\right)\left(( \alpha _ { 7 } + \mu ) \left(\left(\alpha_{7}+\mu\right)\left(4 \mu^{2}\left(\alpha_{7}+\mu\right)-2 B \alpha_{1} \mu\right)\right.\right. \\
& \left.+2 \mu\left(\alpha_{5}+\mu\right)\left(4 \alpha_{5} \mu-B \alpha_{1}\right)+B \alpha_{1}\left(B \alpha_{1}-6 \alpha_{5} \mu\right)+\alpha_{7}^{2} \mu^{2}\right) \\
& \left.+4 \alpha_{5}^{2} \mu\left(-B \alpha_{1}+\alpha_{5}+\mu\right)\right)
\end{aligned}
$$

Eigenvalues $\lambda_{2}^{1}$ and $\lambda_{3}^{1}$ are complex when $\beta_{2}$ is negative, real part of both these eigenvalues are negative and when $\beta_{2}$ is positive, real part of both the eigenvalues $\lambda_{2}^{1}$ and $\lambda_{3}^{1}$ are negative when $\left(\beta_{1}-\sqrt{\beta_{2}}\right)>0$,

i.e $\left(\beta_{1}^{2}-\beta_{2}\right)>0$

$\beta_{1}^{2}-\beta_{2}$

$$
\begin{aligned}
= & \left(\alpha_{7}+\mu\right)\left(( \alpha _ { 7 } + \mu ) \left(\left(\alpha_{7}+\mu\right) 4 \mu\left(B \alpha_{1}-\alpha_{5} \mu-\mu^{2}\right)\right.\right. \\
& \left.\left.+8 \alpha_{5}^{2} \mu\left(B \alpha_{1}-\alpha_{5} \mu-\mu^{2}\right)\right)+4 \alpha_{5}^{2} \mu\left(B \alpha_{1}-\alpha_{5} \mu-\mu^{2}\right)\right)
\end{aligned}
$$

Hence, real part of eigenvalues $\lambda_{2}^{1}$ and $\lambda_{3}^{1}$ are negative when $B \alpha_{1}>\mu\left(\alpha_{5}+\mu\right)$.

$$
\begin{gathered}
\left(( \alpha _ { 7 } + \mu ) \left(\mu\left(\alpha_{4}-\alpha_{2}\right)\left(\alpha_{5}+\mu\right)+\alpha_{1} \mu\left(\mu_{D}+\mu\right)\right.\right. \\
\lambda_{4}^{1}=\frac{\left.-B \alpha_{1} \alpha_{5}+\alpha_{1} \alpha_{6} \mu+\alpha_{1} \alpha_{5} \mu\left(\mu_{D}+\mu\right)-\alpha_{2} \alpha_{5} \mu\left(\alpha_{5}+\mu\right)\right)}{-\alpha_{1} \mu\left(\alpha_{5}+\alpha_{7}+\mu\right)}
\end{gathered}
$$

$\lambda_{4}^{1}<0$ when $\alpha_{2}\left(\alpha_{5}+\mu\right)<\alpha_{1}\left(\mu_{D}+\mu\right)$ and $\alpha_{6} \mu>B \alpha_{5}$

. Clearly, all the eigenvalues are negative under these conditions. Hence, the theorem.

Theorem 3. The malaria free equilibrium point $E_{2}$ is locally asymptotically stable if it satisfy following two conditions.

$$
\text { I. } k_{2}\left(\alpha_{7}+\mu\right) \alpha_{4}+\frac{\alpha_{1} n_{3} k_{2}}{n_{4}}>\alpha_{5}+\mu
$$

II. $\mu>n_{6}, \alpha_{6}>n_{5}$ and $n_{5}^{2}+n_{6}^{2}>2 \mu n_{6}$

Proof. Jacobian matrix of the model around point $E_{2}$ is:

$$
J\left(E_{2}\right)=\left[\begin{array}{cccc}
n_{6}-\mu & -\frac{\alpha_{1} n_{3} k_{2}}{n_{4}} & -n_{5} & \alpha_{7} \\
0 & k_{2}\left(\alpha_{7}+\mu\right) \alpha_{4}+\frac{\alpha_{1} n_{3} k_{2}}{n_{4}}-\alpha_{5}-\mu & 0 & 0 \\
-n_{6} & -k_{2}\left(\alpha_{7}+\mu\right) \alpha_{4} & n_{5}-\mu_{D}-\alpha_{6}-\mu & 0 \\
0 & \alpha_{5} & \alpha_{6} & -\alpha_{7}-\mu
\end{array}\right]
$$

Where, $n_{3}=\mu\left(\mu_{D}+\mu\right)+\alpha_{6} \mu-B \alpha_{2}$,

$$
n_{4}=\left(\alpha_{7}+\mu\right)\left(\left(\mu_{D}+\alpha_{6}\right)\left(B \alpha_{3}+\mu_{D}+\mu\right)\right.
$$$$
\left.+\mu\left(\mu_{D} \mu+B \alpha_{3}+\mu_{D} \alpha_{6}\right)\right)+\alpha_{6} \mu\left(\alpha_{6}+\mu\right),
$$

$$
n_{5}=\frac{-\alpha_{2} n_{2} k_{2}}{n_{4}\left(-k_{2}\left(\alpha_{7}+\mu\right) \alpha_{3}+1\right)^{2}}, n_{6}=\frac{\alpha_{2} k_{2}\left(\alpha_{7}+\mu\right)}{-n_{5}\left(\alpha_{7}+\mu\right) \alpha_{3}+1} \text {. }
$$

Clearly, $\lambda_{1}^{2}=k_{2}\left(\alpha_{7}+\mu\right) \alpha_{4}+\frac{\alpha_{1} n_{3} k_{2}}{n_{4}}-\alpha_{5}-\mu$ is one of the eigen value of $J\left(E_{2}\right)$, hence characteristic equation is given by:

$$
C h(x)=\left(x-k_{2}\left(\alpha_{7}+\mu\right) \alpha_{4}-\frac{\alpha_{1} n_{3} k_{2}}{n_{4}}+\alpha_{5}+\mu\right) \phi(x)
$$

Where, $\phi(x)=x^{3}+a_{1} x^{2}+a_{2} x+a_{3}$ $a_{1}=\alpha_{7}+3 \mu-n_{5}+\mu_{D}+\alpha_{6}-n_{6}$, 


$$
\begin{aligned}
& a_{2}=\left(\alpha_{7}+\mu\right)\left(\alpha_{6}+\mu\right)+\left(\mu-n_{6}\right)\left(\mu_{D}+\alpha_{6}+\alpha_{7}+\mu\right), \\
& \quad+\left(\mu-n_{5}\right)\left(\alpha_{7}+2 \mu\right)-\mu n_{6} \\
& a_{3}=\left(\mu-n_{6}\right)\left(\mu_{D}\left(\alpha_{7}+\mu\right)+\mu\left(\alpha_{6}+\alpha_{7}\right)\right)+\alpha_{7} \mu\left(\alpha_{6}-n_{5}\right) \\
& \quad+\mu^{2}\left(\mu-n_{5}\right) \\
& a_{1} a_{2}-a_{3} \\
& =\left(\alpha_{7}+\mu\right)\left(\mu_{D}\left(\mu_{D} \mu+\alpha_{6}\right)+\left(\alpha_{6}+\alpha_{7}\right)\left(\mu_{D}+\alpha_{6}\right)\right) \\
& +\left(\mu-n_{6}\right)\left(\left(\mu_{D}+2 \mu\right)\left(\mu_{D}+\alpha_{6}\right)+\left(\mu_{D}+\alpha_{6}\right)\left(\alpha_{6}+\alpha_{7}\right)\right. \\
& \left.+\left(\alpha_{7}+\mu\right)\left(\mu_{D}+2 \alpha_{6}+\alpha_{7}\right)+\mu\left(\mu_{D}+\alpha_{7}\right)\right)+\mu_{D} \alpha_{7} \mu \\
& +\left(\mu-n_{5}\right)\left(\left(2 \mu_{D}+2 \alpha_{6}+\alpha_{7}\right)\left(\alpha_{7}+\mu\right)+2 \mu\left(\mu_{D}+\alpha_{6}\right)\right. \\
& \left.+\mu\left(5 \alpha_{7}+8 \mu\right)\right)+n_{6}\left(\mu_{D}+\alpha_{6}\right)\left(n_{5}+n_{6}-2 \mu\right) \\
& +4 \mu n_{6}\left(n_{5}-n_{6}\right)+\left(n_{5}+n_{6}\right)\left(\alpha_{7} n_{5}+\alpha_{7} n_{6}\right) \\
& +2 \mu\left(n_{5}^{2}+n_{6}^{2}-2 \mu n_{6}\right)
\end{aligned}
$$

$a_{1}, a_{3}>0$ and $a_{1} a_{2}>a_{3}$ if $\mu>n_{6}, \alpha_{6}>n_{5}$ and $n_{5}^{2}+n_{6}^{2}>2 \mu n_{6}$. Hence, by applying Routh-Hurwitz criteria we can say all real roots of $C h(x)$ are negative under these conditions.

Theorem 4. The endemic equilibrium point $E^{*}$ is locally asymptotically stable if it satisfy following two conditions.

$$
\begin{aligned}
& \text { I. } k_{2}\left(\alpha_{7}+\mu\right) \alpha_{4}+\frac{\alpha_{1} n_{3} k_{2}}{n_{4}}>\alpha_{5}+\mu \\
& \text { II. } \mu>n_{6}, \alpha_{6}>n_{5} \text { and } n_{5}^{2}+n_{6}^{2}>2 \mu n_{6}
\end{aligned}
$$

Proof. Jacobian matrix of the model around point $E^{*}$ is $J\left(E^{*}\right)=\left[x_{i j}\right]$.

Where, $x_{11}=-\alpha_{1} M^{*}-\frac{\alpha_{2} D^{*}}{D^{*} \alpha_{3}+1}-\mu, x_{12}=-\alpha_{1} S^{*}$,

$x_{13}=\frac{-\alpha_{2} S^{*}}{\left(D^{*} \alpha_{3}+1\right)^{2}}, x_{14}=\alpha_{7}, x_{21}=\alpha_{1} M^{*}$,

$x_{22}=-D^{*} \alpha_{4}+S^{*} \alpha_{1}-\alpha_{5}-\mu, \quad x_{23}=-\alpha_{4} M^{*}, \quad x_{24}=0$, $x_{31}=\frac{\alpha_{2} D^{*}}{D^{*} \alpha_{3}+1}, x_{32}=\alpha_{4} D^{*}, x_{34}=0, x_{41}=0, x_{42}=\alpha_{5}$ $x_{33}=\frac{\alpha_{2} S^{*}}{\left(D^{*} \alpha_{3}+1\right)^{2}}+\alpha_{4} M^{*}-\mu_{D}-\alpha_{6}-\mu, x_{43}=\alpha_{6}$, $x_{44}=-\alpha_{7}-\mu$

The characteristic equation of matrix $J\left(E^{*}\right)$ is $C h^{*}(x)=x^{4}+b_{1} x^{3}+b_{2} x^{2}+b_{3} x+b_{4}=0$.

Where, $b_{1}=-x_{44}-x_{33}-x_{22}-x_{11}$,

$$
\begin{aligned}
b_{2}= & x_{11} x_{22}+x_{11} x_{33}+x_{11} x_{44}-x_{12} x_{21}-x_{13} x_{31}+x_{22} x_{33}, \\
& +x_{22} x_{44}-x_{32} x_{23}+x_{33} x_{44} \\
b_{3}= & -x_{11} x_{22} x_{33}-x_{11} x_{22} x_{44}+x_{11} x_{23} x_{32}-x_{11} x_{33} x_{44} \\
& +x_{12} x_{21} x_{33}+x_{12}\left(x_{21} x_{44}-x_{23} x_{31}\right)-x_{21}\left(x_{13} x_{32}-x_{14} x_{42}\right) \\
& +x_{31} x_{13} x_{22}-x_{31}\left(x_{14} x_{43}-x_{13} x_{44}\right)-x_{22} x_{33} x_{44}+x_{23} x_{32} x_{44} \\
b_{4}= & x_{11} x_{44}\left(x_{22} x_{33}-x_{23} x_{32}\right)+x_{13} x_{44}\left(x_{12} x_{23}-x_{13} x_{22}\right) \\
& +x_{21} x_{32}\left(x_{13} x_{44}-x_{14} x_{43}\right)+x_{21} x_{33}\left(x_{14} x_{42}-x_{12} x_{44}\right) \\
& +x_{14} x_{31}\left(x_{22} x_{43}-x_{23} x_{42}\right) .
\end{aligned}
$$

$b_{2}>0$ when $x_{22}, x_{33}$ are negative.

$x_{22}<0 \Leftrightarrow S^{*} \alpha_{1}<\alpha_{5}+\mu+D^{*} \alpha_{4}$ and

$x_{33}<0 \Leftrightarrow \frac{\alpha_{2} S^{*}}{\left(D^{*} \alpha_{3}+1\right)^{2}}+\alpha_{4} M^{*}<\mu_{D}+\alpha_{6}+\mu$.

$b_{3}>0$ when $\alpha_{1} M^{*}\left(\alpha_{7}-\mu\right)>\frac{\alpha_{4} \alpha_{2} D^{*} M^{*}}{D^{*} \alpha_{3}+1}$,

$\frac{\alpha_{2} \alpha_{4} S^{*} D^{*}}{\left(D^{*} \alpha_{3}+1\right)^{2}}>\alpha_{7} \alpha_{5}$ and $\frac{\alpha_{2} S^{*}\left(\alpha_{7}+\mu\right)}{\left(D^{*} \alpha_{3}+1\right)^{2}}>\alpha_{7} \alpha_{6}$.

$b_{4}=x_{11} x_{44}\left(x_{22} x_{33}-x_{23} x_{32}\right)+x_{13} x_{44}\left(x_{12} x_{23}-x_{13} x_{22}\right)$

$+x_{21} x_{32}\left(x_{13} x_{44}-x_{14} x_{43}\right)+x_{21} x_{33}\left(x_{14} x_{42}-x_{12} x_{44}\right)$

$+x_{14} x_{31}\left(x_{22} x_{43}-x_{23} x_{42}\right)$

and $b_{4}>0$ when,

$\alpha_{1} \alpha_{4} S^{*} M^{*}>\frac{\alpha_{2} S^{*}}{\left(D^{*} \alpha_{3}+1\right)^{2}}\left(D^{*} \alpha_{4}-S^{*} \alpha_{1}+\alpha_{5}+\mu\right)$,

$\frac{\alpha_{2} S^{*}\left(\alpha_{7}+\mu\right)}{\left(D^{*} \alpha_{3}+1\right)^{2}}>\alpha_{7} \alpha_{6}, \alpha_{7} \alpha_{5}>\alpha_{1} S^{*}\left(\alpha_{7}+\mu\right)$,

$\alpha_{4} \alpha_{5} M^{*}>\left(D^{*} \alpha_{4}-S^{*} \alpha_{1}+\alpha_{5}+\mu\right) \alpha_{6}$.

\subsection{Global stability}

To perform the global stability analysis of the disease free equilibrium we use the method developed by [5].

\subsubsection{Global stability of disease-free equilibrium point $\left(E_{0}\right)$}

The model system can be written as follows:

$\frac{d X_{(0)}}{d t}=F_{0}\left(X_{(0)}, Z_{(0)}\right)$
$\frac{d Z_{(0)}}{d t}=G_{0}\left(X_{(0)}, Z_{(0)}\right), G_{0}\left(X_{(0)}, 0\right)=0$

Here $X_{(0)}=X_{(0)}\left(X_{1}^{0}\right) \in \mathbb{R}$ represents the number of uninfected individuals and $Z_{(0)}=Z_{0}\left(Y_{1}^{0}, Y_{2}^{0}, Y_{3}^{0}\right) \in \mathbb{R}^{3}$ denotes the number of infected individuals. According to this notation the disease-free equilibrium point is denoted by $E_{0}=\left(S_{0}, 0\right)$.

Now as per the method given in [5], following two conditions will ensure global stability of the diseasefree equilibrium point.

[H1] $\frac{d X_{(0)}}{d t}=F_{0}\left(X_{(0)}, 0\right), \quad E_{0}=\left(X_{0}, 0\right)$ is globally asymptotically stable.

[H2]

$$
\begin{gathered}
G_{0}\left(X_{(0)}, Z_{(0)}\right)=B_{1} M+\left[B_{2}-\frac{\alpha_{2} \alpha_{3} D S^{0}}{1+\alpha_{3} D}\right] D+B_{3} R-\hat{G}_{0}\left(X_{(0)}, Z_{(0)}\right) \\
\text {, where } \hat{G}_{0}\left(X_{(0)}, Z_{(0)}\right) \geq 0 \text { for }\left(X_{(0)}, Z_{(0)}\right) \in \Lambda .
\end{gathered}
$$

Here, $B_{1}=D_{M} G_{0}\left(X_{0}, 0\right), \quad B_{2}=D_{D} G_{0}\left(X_{0}, 0\right) \quad$ and $B_{3}=D_{R} G_{0}\left(X_{0}, 0\right)$ are matrix with non-negative off diagonal entries.

Lemma 1. The fixed point $E_{0}=\left(S_{0}, 0\right)$ is a globally 
asymptotically stable equilibrium of the system, provided $R_{0}<1$ and assumptions [H1] and [H2] are satisfied.

Theorem 5. For $R_{0}<1$, the disease free equilibrium point is globally asymptotically stable.

Proof. we begin by showing [H1] as $F_{0}\left(X_{(0)}, 0\right)=[B-\mu S]$ and $C h_{(0)}(\lambda)=(\lambda+\mu)=0$ is the characteristic polynomial of its Jacobian matrix. Since the polynomial have a negative root, $E_{0}=\left(S_{0}, 0\right)$ is globally asymptotically stable.

Now, we have

$$
\begin{aligned}
G_{0}\left(X_{(0)}, Z_{(0)}\right)= & \left(\alpha_{1} S_{0}-\mu\right) M+\left(\frac{\alpha_{2} S_{0}}{1+\alpha_{3} D}-\left(\mu+\mu_{D}\right)\right) D-\left(\alpha_{7}+\mu\right) R \\
& -\left(\alpha_{1} M\left(S_{0}-S\right)+\frac{\alpha_{2} D}{1+\alpha_{3} D}-\left(S_{0}-S\right)\right) \\
= & B_{1} M+\left[B_{2}-\frac{\alpha_{2} \alpha_{3} D S_{0}}{1+\alpha_{3} D}\right] D+B_{3} R-\hat{G}_{0}\left(X_{(0)}, Z_{(0)}\right)
\end{aligned}
$$

Here, $\hat{G}_{0}(X, Z) \geq 0$ hence, the conditions (H1) and (H2) stated above are satisfied.

\subsubsection{Global stability of dengue-free equilibrium point $\left(E_{1}\right)$}

The model system can be written as

$$
\begin{aligned}
& \frac{d X_{(1)}}{d t}=F_{1}\left(X_{(1)}, Z_{(1)}\right) \\
& \frac{d Z_{(1)}}{d t}=G_{1}\left(X_{(1)}, Z_{(1)}\right), G_{1}\left(X_{(1)}, 0\right)=0
\end{aligned}
$$

Here $\quad X_{(1)}=X_{(1)}\left(X_{1}^{1}, X_{2}^{1}, X_{3}^{1}\right) \in \mathbb{R}^{3}$ represents the number of uninfected individuals and $Z_{(1)}=Z_{1}\left(Y_{1}^{1}\right) \in \mathbb{R}$ denotes the number of infected individuals. According to this notation the Dengue free equilibrium point is denoted by $E_{1}=\left(X_{(1)}^{1}, 0\right)$, where $X_{(1)}^{1}=\left(S_{1}, M_{1}, R_{1}\right)$.

The following two conditions will ensure global stability of the dengue-free equilibrium point:

[H3] $\frac{d X_{(1)}}{d t}=F_{1}\left(X_{(1)}, 0\right), \quad E_{1}=\left(X_{(1)}^{1}, 0\right)$ is globally asymptotically stable.

[H4] $G_{1}\left(X_{(1)}, Z_{(1)}\right)=B_{4} D-\frac{\alpha_{2} \alpha_{3} D S_{1}}{1+\alpha_{3} D}-\hat{G}_{1}\left(X_{(1)}, Z_{(1)}\right)$, where $\hat{G}_{1}\left(X_{(1)}, Z_{(1)}\right) \geq 0$ for $\left(X_{(1)}, Z_{(1)}\right) \in \Lambda$.

Here $B_{4}=D_{D} G_{1}\left(X_{(1)}^{1}, 0\right)$ is a M-matrix.

Lemma 2. The fixed point $E_{1}=\left(X_{(1)}^{1}, 0\right)$ is a globally asymptotically stable equilibrium of the system, provided $R_{0}<1$ and assumptions [H3] and [H4] are satisfied.

Theorem 6. For $R_{0}<1$, the disease-free equilibrium point is globally asymptotically stable when
$D \alpha_{4}>S \alpha_{1}$.

Proof. we begin by showing [H3] as $F_{1}\left(X_{(1)}, 0\right)=\left[\begin{array}{c}B+\alpha_{7} R-\alpha_{1} S M-\mu S \\ \alpha_{1} S M-\alpha_{5} M-\mu M \\ \alpha_{5} M-\alpha_{7} R-\mu R\end{array}\right]$ and

$C h_{(1)}(\lambda)=\left(\lambda^{3}+c_{1} \lambda^{2}+c_{2} \lambda+c_{3}\right)=0$ is the characteristic polynomial of its Jacobian matrix. Where, $c_{1}=\left(D \alpha_{4}-S \alpha_{1}\right)+M \alpha_{1}+\alpha_{5}+\alpha_{7}+3 \mu$,

$$
\begin{aligned}
& c_{2}=\left(D \alpha_{4}-S \alpha_{1}\right)\left(\alpha_{7}+2 \mu\right)+\left(M \alpha_{1}+\alpha_{7}\right)\left(D \alpha_{4}+\alpha_{5}\right) \\
&+2 \mu\left(M \alpha_{1}+\alpha_{5}\right)+2 \mu\left(\alpha_{7}+\mu\right)+\mu^{2} \\
& c_{3}=\left(\alpha_{7}+\mu\right)\left(\mu\left(D \alpha_{4}-S \alpha_{1}\right)+M D \alpha_{1} \alpha_{4}+\alpha_{5} \mu\right) \\
&+M \alpha_{1} \mu\left(\alpha_{5}+\alpha_{7}\right)+\mu^{2}\left(M \alpha_{1}+\alpha_{7}\right)+\mu^{3} . \\
& c_{1} c_{2}-c_{3}=\left(D \alpha_{4}-S \alpha_{1}\right) \\
&\left(\left(\alpha_{7}+\mu\right)\left(D \alpha_{4}+2 M \alpha_{1}+2 \alpha_{5}+\alpha_{7}+4 \mu\right)\right. \\
&+\left.M \alpha_{1}\left(D \alpha_{4}+\alpha_{5}\right)\right)+\left(\alpha_{7}+2 \mu\right)\left(M \alpha_{1}\left(3 \alpha_{5}+M \alpha_{1}\right)\right. \\
&+\left.\left(\alpha_{7}+4 \mu\right)\left(M \alpha_{1}+\alpha_{5}\right)+2 \mu\left(\alpha_{7}+2 \mu\right)+\alpha_{5}^{2}\right) \\
&+ M \alpha_{1}^{2}\left(D \alpha_{4}+\alpha_{5}\right)+S^{2} \alpha_{1}^{2}\left(\alpha_{7}+\mu\right) \\
&+ M \alpha_{1} \alpha_{5}\left(\alpha_{5}+D \alpha_{4}\right)+2 D M \alpha_{1} \alpha_{4} \mu \\
& c_{1}, c_{2}>0 \text { and } c_{1} c_{2}-c_{3}>0 \text { if } D \alpha_{4}>S \alpha_{1} .
\end{aligned}
$$

With the help of Routh-Hurwitz criteria it is clear that all the roots of the characteristic polynomial have negative real part when $D \alpha_{4}>S \alpha_{1}$, hence $E_{1}=\left(X_{(1)}^{1}, 0\right)$ is globally asymptotically stable under this condition.

Now,

$$
\begin{aligned}
G_{1}\left(X_{(1)}, Z_{(1)}\right) & =\left(\frac{\alpha_{2} S_{1}}{1+\alpha_{3} D}+\alpha_{4} M_{1}-\alpha_{6}-\left(\mu+\mu_{D}\right)\right) D \\
& -\left(\frac{\alpha_{2} D}{1+\alpha_{3} D}\left(S_{1}-S\right)+\alpha_{4} D\left(M_{1}-M\right)\right) \\
& =\left(B_{5}-\frac{\alpha_{2} \alpha_{3} D S_{1}}{1+\alpha_{3} D}\right) D-\hat{G}_{1}\left(X_{(1)}, Z_{(1)}\right)
\end{aligned}
$$

Here, $\hat{G}_{1}(X, Z) \geq 0$ hence, the conditions (H3) and (H4) stated above are satisfied.

\subsubsection{Global stability of malaria-free equilibrium point $\left(E_{2}\right)$}

The model system can be written as:

$$
\begin{aligned}
& \frac{d X_{(2)}}{d t}=F_{2}\left(X_{(2)}, Z_{(2)}\right) \\
& \frac{d Z_{(2)}}{d t}=G_{2}\left(X_{(2)}, Z_{(2)}\right), G_{2}\left(X_{(2)}, 0\right)=0
\end{aligned}
$$

Here $\quad X_{(2)}=X_{(2)}\left(X_{1}^{2}, X_{2}^{2}, X_{3}^{2}\right) \in \mathbb{R}^{3}$ represents the number of uninfected individuals and $Z_{(2)}=Z_{2}\left(Y_{1}^{2}\right) \in \mathbb{R}$ denotes the number of infected individuals. According to this notation the Dengue free equilibrium point is denoted by $E_{2}=\left(X_{(2)}^{1}, 0\right)$, where 


$$
X_{(2)}^{1}=\left(S_{2}, D_{2}, R_{2}\right) \text {. }
$$

The following two conditions will ensure global stability of the malaria-free equilibrium point:

[H5] $\frac{d X_{(2)}}{d t}=F_{2}\left(X_{(2)}, 0\right), \quad E_{2}=\left(X_{(2)}^{1}, 0\right)$ is globally asymptotically stable.

[H6] $G_{2}\left(X_{(2)}, Z_{(2)}\right)=B_{5} M-\hat{G}_{2}\left(X_{(2)}, Z_{(2)}\right)$, where

$$
\hat{G}_{2}\left(X_{(2)}, Z_{(2)}\right) \geq 0 \text { for }\left(X_{(2)}, Z_{(2)}\right) \in \Lambda \text {. }
$$

Here $B_{5}=D_{M} G_{2}\left(X_{(2)}^{1}, 0\right)$ is a M-matrix.

Lemma 3. The fixed point $E_{2}=\left(X_{(2)}^{1}, 0\right)$ is a globally asymptotically stable equilibrium of the system, provided $R_{0}<1$ and assumptions [H5] and [H6] are satisfied.

Theorem 7. For $R_{0}<1$, the disease-free equilibrium point is globally asymptotically stable if $\frac{\alpha_{2} S}{\left(1+\alpha_{3} D\right)^{2}}<\alpha_{6}+\mu+\mu_{D}$.

Proof. we begin by showing [H5] as:

$$
F_{2}\left(X_{(2)}, 0\right)=\left[\begin{array}{c}
B+\alpha_{7} R-\frac{\alpha_{2} S D}{1+\alpha_{3} D}-\mu S \\
\frac{\alpha_{2} S D}{1+\alpha_{3} D}-\alpha_{6} D-\left(\mu+\mu_{D}\right) D \\
\alpha_{6} D-\alpha_{7} R-\mu R
\end{array}\right] \text { and }
$$

$C h_{(2)}(\lambda)=\left(\lambda^{3}+d_{1} \lambda^{2}+d_{2} \lambda+d_{3}\right)=0$ is the

characteristic polynomial of its Jacobian matrix.

Where, $d_{1}=n_{7}+n_{8}+n_{9}$,

$d_{2}=\left(n_{9}+\mu\right)\left(n_{7}+n_{8}\right)+n_{7}\left(\mu_{D}+\alpha_{6}\right)-\mu\left(\mu_{D}+\alpha_{6}+\mu\right)$

and $d_{3}=\left(n_{7}-\mu\right)\left(\mu_{D} n_{9}+n_{9} \mu+\alpha_{6} \mu\right)+n_{8} n_{9} \mu$

where, $n_{7}=\frac{\alpha_{2} D}{1+\alpha_{3} D}+\mu$,

$$
\begin{aligned}
& n_{8}=\frac{-\alpha_{2} S}{\left(1+\alpha_{3} D\right)^{2}}+\alpha_{6}+\mu+\mu_{D} \text { and } n_{9}=\alpha_{7}+\mu \text {. } \\
& d_{1} d_{2}-d_{3}=\left(n_{7}-\mu\right) \\
& \left(\left(n_{7}+n_{8}\right)\left(\mu_{D}+\mu+\alpha_{6}\right)+\alpha_{6}\left(n_{9}+\mu\right)\right) \\
& +n_{7} n_{8}\left(n_{9}+\mu\right)+n_{8} n_{9}\left(n_{7}+n_{8}\right) \\
& +n_{7} n_{9}\left(n_{7}+n_{9}\right)+n_{8}\left(n_{8} \mu+n_{9}^{2}\right)
\end{aligned}
$$

Clearly, $n_{7}>\mu$ hence $d_{3}>0$ and $d_{1} d_{2}-d_{3}>0 . d_{1}>0$ if $\frac{\alpha_{2} S}{\left(1+\alpha_{3} D\right)^{2}}<\alpha_{6}+\mu+\mu_{D}$.

With the help of Routh-Hurwitz criteria it is clear that all the roots of the characteristic polynomial have negative real part, hence $E_{2}=\left(X_{(2)}^{1}, 0\right)$ is globally asymptotically stable.

Now,

$$
\begin{aligned}
G_{2}\left(X_{(2)}, Z_{(2)}\right)= & \left(\alpha_{1} S_{2}-\alpha_{4} D_{2}-\alpha_{5}-\mu\right) M \\
& -\left(\alpha_{1}\left(S_{2}-S\right)+\alpha_{4}\left(D_{2}-D\right)\right) M \\
= & B_{5} M-\hat{G}_{2}\left(X_{(2)}, Z_{(2)}\right)
\end{aligned}
$$

Here, $\hat{G}_{2}(X, Z) \geq 0$ hence, the conditions (H5) and (H6) stated above are satisfied.

\subsubsection{Global stability of endemic equilibrium point $\left(\boldsymbol{E}^{*}\right)$}

We analyze global stability of an endemic equilibrium point through a geometric approach described in [16], [27] and [15]. To use this method let we modify our system (1) as follow:

$$
\begin{aligned}
& \frac{d S}{d t}=B+\frac{\alpha_{7}\left(\alpha_{6} D+\alpha_{5} M\right)}{\left(\alpha_{7}+\mu\right)}-\alpha_{1} S M-\frac{\alpha_{2} S D}{1+\alpha_{3} D}-\mu S \\
& \frac{d M}{d t}=\alpha_{1} S M-\alpha_{4} M D-\alpha_{5} M-\mu M
\end{aligned}
$$

$\frac{d D}{d t}=\frac{\alpha_{2} S D}{1+\alpha_{3} D}+\alpha_{4} M D-\alpha_{6} D-\left(\mu+\mu_{D}\right) D$

Let $K \subset \mathbb{R}^{3}$ be a simply connected open set and $f \in C^{1}(K)$. Further suppose that $\varphi(t)$ be a solution to the following system,

$x^{\prime}=f(x)$

(7)Suppose $P(x)$ be a matrix valued function on $K$ and let $Q=P_{f} P^{-1}+P M^{[2]} P^{-1}$.

Here, the matrix $P_{f}$ is:

$$
\left(P_{i j}(x)\right)_{f}=\left(\frac{\partial P_{i j}(x)}{\partial x}\right) \cdot f(x)=\nabla P_{i j} \cdot f(x) .
$$

Jacobian matrix of an arbitrary point is $M=\left[c_{i j}\right]$ :

Where, $c_{11}=-\alpha_{1} M-\frac{\alpha_{2} D}{1+\alpha_{3} D}-\mu, c_{12}=\frac{\alpha_{7} \alpha_{5}}{\alpha_{7}+\mu}-\alpha_{1} S$

$c_{13}=\frac{\alpha_{7} \alpha_{6}}{\alpha_{7}+\mu}-\frac{\alpha_{2} S}{\left(1+\alpha_{3} D\right)^{2}}, \quad c_{21}=\alpha_{1} M, c_{23}=-\alpha_{4} M$,

$c_{22}=\alpha_{1} S-\alpha_{4} D-\alpha_{5}-\mu, \quad c_{31}=\frac{\alpha_{2} D}{1+\alpha_{3} D}, \quad c_{32}=\alpha_{4}$,

$c_{33}=\frac{\alpha_{2} S}{\left(1+\alpha_{3} D\right)^{2}}+\alpha_{4} M-\alpha_{6}-\left(\mu+\mu_{D}\right)$.

The second additive compound matrix obtain from the Jacobian matrix $M$ is $M^{[2]}$,

$$
M^{[2]}=\left[\begin{array}{ccc}
n_{10} & -\alpha_{4} M & \frac{\alpha_{2} S}{\left(1+\alpha_{3} D\right)^{2}}-\frac{\alpha_{7} \alpha_{6}}{\alpha_{7}+\mu} \\
\alpha_{4} & n_{11} & \frac{\alpha_{7} \alpha_{5}}{\alpha_{7}+\mu}-\alpha_{1} S \\
-\frac{\alpha_{2} D}{1+\alpha_{3} D} & \alpha_{1} M & n_{12}
\end{array}\right]
$$

Where $n_{10}=\alpha_{1} S-\alpha_{4} D-\alpha_{5}-2 \mu-\alpha_{1} M-\frac{\alpha_{2} D}{1+\alpha_{3} D}$, 
$n_{11}=\frac{\alpha_{2} S}{\left(1+\alpha_{3} D\right)^{2}}+\alpha_{4} M-\alpha_{6}-2 \mu-\mu_{D}-\alpha_{1} M-\frac{\alpha_{2} D}{1+\alpha_{3} D}$ , and

$n_{12}=\alpha_{1} S-\alpha_{4} D-\alpha_{5}+\frac{\alpha_{2} S}{\left(1+\alpha_{3} D\right)^{2}}+\alpha_{4} M-\alpha_{6}-2 \mu-\mu_{D}$

Next, consider the following system:

$\frac{d z}{d t}=\Phi(\varphi(t)) z$

And if (8) is stable then also the second compound equation $\frac{d \bar{z}}{d t}=M^{[2]}(\varphi(t)) \bar{z}$ is stable, moreover $\varphi$ belong to a set in which $\left|P^{-1}\right|$ is bounded. A set $\bar{K}$ is absorbing with respect to (7) if solution exist for all $t \geq 0$ and $x\left(t, K_{1}\right) \subset \bar{K}$ for all $t$, where $K_{1}$ is any bounded subset of $K$.

To prove global stability through this approach we use techniques developed in [19].

Let, $P=\frac{1}{D} I_{3}$, where $I_{3}$ is an identity matrix of order

3. Hence, $P_{f} P^{-1}=-\frac{D^{\prime}}{D} I_{3}$.

Next, $Q=P_{f} P^{-1}+P M^{[2]} P^{-1}$

$$
=\left[\begin{array}{ccc}
n_{10}-\frac{D^{\prime}}{D} & -\alpha_{4} M & \frac{\alpha_{2} S}{\left(1+D \alpha_{3}\right)^{2}}-\frac{\alpha_{7} \alpha_{6}}{\alpha_{7}+\mu} \\
\alpha_{4} & n_{11}-\frac{D^{\prime}}{D} & \frac{\alpha_{7} \alpha_{5}}{\alpha_{7}+\mu}-\alpha_{1} S \\
-\frac{\alpha_{2} D}{1+\alpha_{3} D} & \alpha_{1} M & n_{12}-\frac{D^{\prime}}{D}
\end{array}\right]
$$

Where,

$n_{10}-\frac{D^{\prime}}{D}=\alpha_{1} S-\alpha_{4} D-\alpha_{5}-\mu-\left(\alpha_{1}+\alpha_{4}\right) M-\frac{\alpha_{2}(S+D)}{1+\alpha_{3} D}$

$n_{11}-\frac{D^{\prime}}{D}=\frac{\alpha_{3} \alpha_{2} S D}{\left(1+D \alpha_{3}\right)^{2}}-\alpha_{1} S-\frac{\alpha_{2} D}{1+\alpha_{3} D}-\mu$ and

$n_{12}-\frac{D^{\prime}}{D}=\frac{-\alpha_{3} \alpha_{2} S D}{\left(1+D \alpha_{3}\right)^{2}}+\alpha_{1} S-\alpha_{4} D-\alpha_{5}-\mu$.

Let we define the following norm function as described in [2] for some $z=\left(z_{1}, z_{2}, z_{3}\right)$.

$$
\|z\|= \begin{cases}\max \left\{\left|z_{1}\right|+\left|z_{3}\right|,\left|z_{2}\right|+\left|z_{3}\right|\right\} & \text { if } z_{2} z_{3} \geq 0 \\ \max \left\{\left|z_{1}\right|+\left|z_{3}\right|,\left|z_{2}\right|\right\} & \text { if } z_{2} z_{3}<0\end{cases}
$$

Now we explore the existence of some $\chi>0$, so that $D_{+}\|z\| \leq-\chi\|z\|$. In this situation we have to analyses all eight possible cases.

Case 1 If $0<z_{1}, z_{2}, z_{3}$ and $\left|z_{1}\right|+\left|z_{3}\right|>\left|z_{2}\right|+\left|z_{3}\right|$ then $\|z\|=\left|z_{1}\right|+\left|z_{3}\right|$ and $D_{+}\|z\|=D_{+}\left(\left|z_{1}\right|+\left|z_{3}\right|\right)=z_{1}^{\prime}+z_{3}^{\prime}$
$D_{+}\|z\|=\left[\alpha_{1} S-\alpha_{4} D-\alpha_{5}-\mu-\left(\alpha_{1}+\alpha_{4}\right) M\right.$

$$
\begin{aligned}
& \left.-\frac{\alpha_{2}(S+D)}{1+\alpha_{3} D}-\alpha_{6}-\mu_{D}\right] z_{1}+\left[-\alpha_{4} M\right] z_{2} \\
& +\left[\frac{\alpha_{2} S}{\left(D \alpha_{3}+1\right)^{2}}-\frac{\alpha_{7} \alpha_{6}}{\alpha_{7}+\mu}\right] z_{3}+\left[-\frac{\alpha_{2} D}{D \alpha_{3}+1}\right] z_{1} \\
& +\left[\alpha_{1} M\right] z_{2} \\
& +\left[\frac{-\alpha_{2} \alpha_{3} S D}{\left(D \alpha_{3}+1\right)^{2}}+\alpha_{1} S-\alpha_{4} D-\alpha_{5}-\mu\right] z_{3} \\
& \leq\left[\alpha_{1} S-\alpha_{4} D-\alpha_{5}-\mu-\left(\alpha_{1}+\alpha_{4}\right) M-\frac{\alpha_{2}(S+D)}{1+\alpha_{3} D}\right. \\
& \left.-\alpha_{6}-\mu_{D}\right]\left|z_{1}\right|+\left[\alpha_{1} M-\alpha_{4} M\right]\left|z_{2}\right| \\
& +\left[\frac{\alpha_{2} S\left(1-\alpha_{3} D\right)}{\left(D \alpha_{3}+1\right)^{2}}-\frac{\alpha_{7} \alpha_{6}}{\alpha_{7}+\mu}+\alpha_{1} S-\alpha_{4} D\right. \\
& \left.-\alpha_{5}-\mu\right]\left|z_{3}\right|
\end{aligned}
$$

Since $\left|z_{1}\right|>\left|z_{2}\right|$, we get

$$
\begin{aligned}
\leq & {\left[\alpha_{1} S-\alpha_{4} D-\alpha_{5}-\mu-\alpha_{4} M-\frac{\alpha_{2}(S+D)}{1+\alpha_{3} D}\right.} \\
& \left.-\alpha_{6}-\mu_{D}\right]\left|z_{1}\right|+\left[\frac{\alpha_{2} S\left(1-\alpha_{3} D\right)}{\left(D \alpha_{3}+1\right)^{2}}-\frac{\alpha_{7} \alpha_{6}}{\alpha_{7}+\mu}\right. \\
& \left.+\alpha_{1} S-\alpha_{4} D-\alpha_{5}-\mu\right]\left|z_{3}\right| \\
D_{+}\|z\| \leq & \max \left\{\alpha_{1} S-\alpha_{4} D-\alpha_{5}-\mu-\alpha_{4} M-\frac{\alpha_{2}(S+D)}{1+\alpha_{3} D}\right. \\
& -\alpha_{6}-\mu_{D} \frac{\alpha_{2} S\left(1-\alpha_{3} D\right)}{\left(D \alpha_{3}+1\right)^{2}}-\frac{\alpha_{7} \alpha_{6}}{\alpha_{7}+\mu}+\alpha_{1} S-\alpha_{4} D \\
& \left.-\alpha_{5}-\mu\right\}\|z\|
\end{aligned}
$$

- ase $_{\boldsymbol{a}} \boldsymbol{q}_{D}$ If $\quad 0<z_{1}, z_{2}, z_{3}$ and $\left|z_{1}\right|+\left|z_{3}\right|<\left|z_{2}\right|+\left|z_{3}\right|$ then $\|z\|=\left|z_{2}\right|+\left|z_{3}\right|$ and $D_{+}\|z\|=z_{2}^{\prime}+z_{3}^{\prime}$ $D_{+}\|z\|=\left[\alpha_{4}\right] z_{1}+\left[-\alpha_{1} M-\frac{\alpha_{2} \alpha_{3} S D}{1+\alpha_{3} D}-\frac{\alpha_{2} D}{D \alpha_{3}+1}-\mu\right] z_{2}$ $+\left[\frac{\alpha_{7} \alpha_{5}}{\alpha_{7}+\mu}-\alpha_{1} S\right] z_{3}+\left[-\frac{\alpha_{2} D}{D \alpha_{3}+1}\right] z_{1}+\left[\alpha_{1} M\right] z_{2}$ $+\left[\frac{-\alpha_{2} \alpha_{3} S D}{\left(D \alpha_{3}+1\right)^{2}}+\alpha_{1} S-\alpha_{4} D-\alpha_{5}-\mu\right] z_{3}$ $\leq\left[\alpha_{4}-\frac{\alpha_{2} D}{D \alpha_{3}+1}\right]\left|z_{1}\right|+\left[-\frac{\alpha_{2} \alpha_{3} S D}{1+\alpha_{3} D}-\frac{\alpha_{2} D}{D \alpha_{3}+1}-\mu\right]\left|z_{2}\right|$ $+\left[\frac{\alpha_{7} \alpha_{5}}{\alpha_{7}+\mu}-\frac{\alpha_{2} \alpha_{3} S D}{\left(D \alpha_{3}+1\right)^{2}}-\alpha_{4} D-\alpha_{5}-\mu\right]\left|z_{3}\right|$ 
Since $\left|z_{1}\right|<\left|z_{2}\right|$, we get

$$
\begin{aligned}
D_{+}\|z\| & \leq\left[\alpha_{4}-\frac{\alpha_{2} D}{D \alpha_{3}+1}-\frac{\alpha_{2} \alpha_{3} S D}{1+\alpha_{3} D}-\mu\right]\left|z_{2}\right| \\
& +\left[\frac{\alpha_{7} \alpha_{5}}{\alpha_{7}+\mu}-\frac{\alpha_{2} \alpha_{3} S D}{\left(D \alpha_{3}+1\right)^{2}}-\alpha_{4} D-\alpha_{5}-\mu\right]\left|z_{3}\right|
\end{aligned}
$$

$D_{+}\|z\| \leq \max \left\{\alpha_{4}-\frac{\alpha_{2} D}{D \alpha_{3}+1}-\frac{\alpha_{2} \alpha_{3} S D}{1+\alpha_{3} D}-\mu, \frac{\alpha_{7} \alpha_{5}}{\alpha_{7}+\mu}\right.$

$$
\left.-\frac{\alpha_{2} \alpha_{3} S D}{\left(D \alpha_{3}+1\right)^{2}}-\alpha_{4} D-\alpha_{5}-\mu\right\}\|z\|
$$

Case 3 If $z_{1}<0<z_{2}, z_{3}$ and $\left|z_{1}\right|+\left|z_{3}\right|>\left|z_{2}\right|+\left|z_{3}\right|$ then $\|z\|=\left|z_{1}\right|+\left|z_{3}\right|$ and $D_{+}\|z\|=-z_{1}^{\prime}+z_{3}^{\prime}$ $D_{+}\|z\|=\left[-\alpha_{1} S+\alpha_{4} D+\alpha_{5}+\mu+\left(\alpha_{1}+\alpha_{4}\right) M\right.$

$$
\begin{aligned}
& \left.+\frac{\alpha_{2}(S+D)}{1+\alpha_{3} D}+\alpha_{6}+\mu_{D}\right] z_{1}+\left[\alpha_{4} M\right] z_{2} \\
& +\left[-\frac{\alpha_{2} S}{\left(D \alpha_{3}+1\right)^{2}}+\frac{\alpha_{7} \alpha_{6}}{\alpha_{7}+\mu}\right] z_{3}+\left[-\frac{\alpha_{2} D}{D \alpha_{3}+1}\right] z_{1} \\
& +\left[\alpha_{1} M\right] z_{2}+\left[\begin{array}{l}
\frac{-\alpha_{2} \alpha_{3} S D}{\left(D \alpha_{3}+1\right)^{2}}+\alpha_{1} S-\alpha_{4} D \\
-\alpha_{5}-\mu
\end{array}\right] z_{3} \\
& \leq\left[-\alpha_{1} S+\alpha_{4} D+\alpha_{5}+\mu+\left(\alpha_{1}+\alpha_{4}\right) M\right. \\
& \left.+\frac{\alpha_{2} S}{1+\alpha_{3} D}+\alpha_{6}+\mu_{D}\right]\left|z_{1}\right|+\left[\alpha_{1} M+\alpha_{4} M\right]\left|z_{2}\right| \\
& +\left[\begin{array}{l}
\frac{\alpha_{7} \alpha_{6}}{\alpha_{7}+\mu}-\frac{\alpha_{2} S\left(1+\alpha_{3} D\right)}{\left(D \alpha_{3}+1\right)^{2}}+\alpha_{1} S-\alpha_{4} D \\
-\alpha_{5}-\mu
\end{array}\right]\left|z_{3}\right|
\end{aligned}
$$

Since $\left|z_{1}\right|>\left|z_{2}\right|$, we get

$$
\begin{aligned}
& \leq\left[\begin{array}{l}
-\alpha_{1} S+\alpha_{4} D+\alpha_{5}+\mu+\left(2 \alpha_{1}+\alpha_{4}\right) M \\
+\alpha_{4} M+\frac{\alpha_{2} S}{1+\alpha_{3} D}+\alpha_{6}+\mu_{D}
\end{array}\right]\left|z_{1}\right| \\
& +\left[\begin{array}{l}
\frac{\alpha_{7} \alpha_{6}}{\alpha_{7}+\mu}-\frac{\alpha_{2} S\left(1+\alpha_{3} D\right)}{\left(D \alpha_{3}+1\right)^{2}}+\alpha_{1} S \\
-\alpha_{4} D-\alpha_{5}-\mu
\end{array}\right]\left|z_{3}\right|
\end{aligned}
$$

$D_{+}\|z\| \leq \max \|z\|\left\{-\alpha_{1} S+\alpha_{4} D+\alpha_{5}+\mu+\left(2 \alpha_{1}+\alpha_{4}\right) M\right.$

$$
\begin{aligned}
& +\alpha_{4} M+\frac{\alpha_{2} S}{1+\alpha_{3} D}+\alpha_{6}+\mu_{D}, \\
& \left.\frac{\alpha_{7} \alpha_{6}}{\alpha_{7}+\mu}-\frac{\alpha_{2} S\left(1+\alpha_{3} D\right)}{\left(D \alpha_{3}+1\right)^{2}}+\alpha_{1} S-\alpha_{4} D-\alpha_{5}-\mu\right\}\|z\|
\end{aligned}
$$

Case 4 If $z_{1}<0<z_{2}, z_{3}$ and $\left|z_{1}\right|+\left|z_{3}\right|<\left|z_{2}\right|+\left|z_{3}\right|$ then $\|z\|=\left|z_{2}\right|+\left|z_{3}\right|$ and $D_{+}\|z\|=z_{2}^{\prime}+z_{3}^{\prime}$ $D_{+}\|z\|=\left[\alpha_{4}\right] z_{1}+\left[-\alpha_{1} M-\frac{\alpha_{2} \alpha_{3} S D}{1+\alpha_{3} D}-\frac{\alpha_{2} D}{D \alpha_{3}+1}-\mu\right] z_{2}$

$$
\begin{aligned}
& +\left[\frac{\alpha_{7} \alpha_{5}}{\alpha_{7}+\mu}-\alpha_{1} S\right] z_{3}+\left[-\frac{\alpha_{2} D}{D \alpha_{3}+1}\right] z_{1}+\left[\alpha_{1} M\right] z_{2} \\
& +\left[\frac{-\alpha_{2} \alpha_{3} S D}{\left(D \alpha_{3}+1\right)^{2}}+\alpha_{1} S-\alpha_{4} D-\alpha_{5}-\mu\right] z_{3} \\
& \leq\left[\alpha_{4}-\frac{\alpha_{2} D}{D \alpha_{3}+1}\right]\left|z_{1}\right| \\
& +\left[-\frac{\alpha_{2} \alpha_{3} S D}{1+\alpha_{3} D}-\frac{\alpha_{2} D}{D \alpha_{3}+1}-\mu\right]\left|z_{2}\right| \\
& +\left[\frac{\alpha_{7} \alpha_{5}}{\alpha_{7}+\mu}-\frac{\alpha_{2} \alpha_{3} S D}{\left(D \alpha_{3}+1\right)^{2}}-\alpha_{4} D-\alpha_{5}-\mu\right]\left|z_{3}\right|
\end{aligned}
$$

Since $\left|z_{1}\right|<\left|z_{2}\right|$, we get

$$
\begin{aligned}
& D_{+}\|z\| \leq {\left[\alpha_{4}-\frac{\alpha_{2} D}{D \alpha_{3}+1}-\frac{\alpha_{2} \alpha_{3} S D}{1+\alpha_{3} D}-\mu\right]\left|z_{2}\right| } \\
&+ {\left[\frac{\alpha_{7} \alpha_{5}}{\alpha_{7}+\mu}-\frac{\alpha_{2} \alpha_{3} S D}{\left(D \alpha_{3}+1\right)^{2}}-\alpha_{4} D-\alpha_{5}-\mu\right]\left|z_{3}\right| } \\
& D_{+}\|z\| \leq \max \left\{\alpha_{4}-\frac{\alpha_{2} D}{D \alpha_{3}+1}-\frac{\alpha_{2} \alpha_{3} S D}{1+\alpha_{3} D}-\mu, \frac{\alpha_{7} \alpha_{5}}{\alpha_{7}+\mu}\right. \\
&\left.\quad-\frac{\alpha_{2} \alpha_{3} S D}{\left(D \alpha_{3}+1\right)^{2}}-\alpha_{4} D-\alpha_{5}-\mu\right\}\|z\|
\end{aligned}
$$

Case 5 If $z_{2}<0<z_{1}, z_{3}$ and $\left|z_{1}\right|+\left|z_{3}\right|>\left|z_{2}\right|$, then $\|z\|=\left|z_{1}\right|+\left|z_{3}\right|$ and $D_{+}\|z\|=z_{1}^{\prime}+z_{3}^{\prime}$

$$
\begin{aligned}
& D_{+}\|z\|=\left[\begin{array}{l}
\alpha_{1} S-\alpha_{4} D-\alpha_{5}-\mu-\left(\alpha_{1}+\alpha_{4}\right) M \\
-\frac{\alpha_{2}(S+D)}{1+\alpha_{3} D}-\alpha_{6}-\mu_{D}
\end{array}\right] z_{1} \\
& +\left[-\alpha_{4} M\right] z_{2}+\left[\frac{\alpha_{2} S}{\left(D \alpha_{3}+1\right)^{2}}-\frac{\alpha_{7} \alpha_{6}}{\alpha_{7}+\mu}\right] z_{3} \\
& +\left[-\frac{\alpha_{2} D}{D \alpha_{3}+1}\right] z_{1}+\left[\alpha_{1} M\right] z_{2} \\
& +\left[\frac{-\alpha_{2} \alpha_{3} S D}{\left(D \alpha_{3}+1\right)^{2}}+\alpha_{1} S-\alpha_{4} D-\alpha_{5}-\mu\right] z_{3} \\
& \leq\left[\begin{array}{l}
\alpha_{1} S-\alpha_{4} D-\alpha_{5}-\mu-\left(\alpha_{1}+\alpha_{4}\right) M \\
-\frac{\alpha_{2}(S+D)}{1+\alpha_{3} D}-\alpha_{6}-\mu_{D}
\end{array}\right] \mid z_{1} \\
& +\left[\alpha_{1} M-\alpha_{4} M\right]\left|z_{2}\right|+\left[\frac{\alpha_{2} S\left(1-\alpha_{3} D\right)}{\left(D \alpha_{3}+1\right)^{2}}\right. \\
& \left.-\frac{\alpha_{7} \alpha_{6}}{\alpha_{7}+\mu}+\alpha_{1} S-\alpha_{4} D-\alpha_{5}-\mu\right]\left|z_{3}\right|
\end{aligned}
$$


Since $\left|z_{1}\right|+\left|z_{3}\right|>\left|z_{2}\right|$, we get

$$
\begin{aligned}
& \leq\left[\begin{array}{l}
\alpha_{1} S-\alpha_{4} D-\alpha_{5}-\mu-\alpha_{4} M-\frac{\alpha_{2}(S+D)}{1+\alpha_{3} D} \\
-\alpha_{6}-\mu_{D}
\end{array}\right]\left|z_{1}\right| \\
& +\left[\begin{array}{l}
\frac{\alpha_{2} S\left(1-\alpha_{3} D\right)}{\left(D \alpha_{3}+1\right)^{2}}-\frac{\alpha_{7} \alpha_{6}}{\alpha_{7}+\mu}+\alpha_{1}(S+M) \\
-\alpha_{4}(D+M)-\alpha_{5}-\mu
\end{array}\right]\left|z_{3}\right| \\
& D_{+}\|z\| \leq \max \left\{\alpha_{1} S-\alpha_{4} D-\alpha_{5}-\mu-\alpha_{4} M-\frac{\alpha_{2}(S+D)}{1+\alpha_{3} D}\right. \\
& -\alpha_{6}-\mu_{D}, \frac{\alpha_{2} S\left(1-\alpha_{3} D\right)}{\left(D \alpha_{3}+1\right)^{2}}-\frac{\alpha_{7} \alpha_{6}}{\alpha_{7}+\mu}+\alpha_{1}(S+M) \\
& \left.-\alpha_{4}(D+M)-\alpha_{5}-\mu\right\}\|z\|
\end{aligned}
$$

Case 6 If $z_{2}<0<z_{1}, z_{3}$ and $\left|z_{1}\right|+\left|z_{3}\right|<\left|z_{2}\right|$ then $\|z\|=\left|z_{2}\right|$ and

$D_{+}\|z\|=-z_{2}^{\prime}=\left[-\alpha_{4}\right] z_{1}$

$$
\begin{aligned}
& +\left[\alpha_{1} M+\frac{\alpha_{2} \alpha_{3} S D}{1+\alpha_{3} D}+\frac{\alpha_{2} D}{D \alpha_{3}+1}+\mu\right] z_{2} \\
& +\left[\alpha_{1} S-\frac{\alpha_{7} \alpha_{5}}{\alpha_{7}+\mu}\right] z_{3}
\end{aligned}
$$

Since $\left|z_{1}\right|+\left|z_{3}\right|<\left|z_{2}\right|$, we get

$$
D_{+}\|z\| \leq\left[\begin{array}{l}
\frac{\alpha_{2} D}{D \alpha_{3}+1}+\frac{\alpha_{2} \alpha_{3} S D}{1+\alpha_{3} D}-\frac{\alpha_{7} \alpha_{5}}{\alpha_{7}+\mu} \\
+\alpha_{1}(M+S)-\alpha_{4}+\mu
\end{array}\right]\|z\|
$$

Case 7 If $z_{3}<0<z_{1}, z_{2}$ and $\left|z_{1}\right|+\left|z_{3}\right|>\left|z_{2}\right|$ then $\|z\|=\left|z_{1}\right|+\left|z_{3}\right|$ and

$$
\begin{aligned}
D_{+}\|z\| & =z_{1}^{\prime}-z_{3}^{\prime}=\left[\begin{array}{l}
\alpha_{1} S-\alpha_{4} D-\alpha_{5}-\mu-\left(\alpha_{1}+\alpha_{4}\right) M \\
-\frac{\alpha_{2}(S+D)}{1+\alpha_{3} D}-\alpha_{6}-\mu_{D}
\end{array}\right] z_{1} \\
& +\left[-\alpha_{4} M\right] z_{2}+\left[\frac{\alpha_{2} S}{\left(D \alpha_{3}+1\right)^{2}}-\frac{\alpha_{7} \alpha_{6}}{\alpha_{7}+\mu}\right] z_{3} \\
& +\left[\frac{\alpha_{2} D}{D \alpha_{3}+1}\right] z_{1}+\left[-\alpha_{1} M\right] z_{2} \\
& +\left[\frac{\alpha_{2} \alpha_{3} S D}{\left(D \alpha_{3}+1\right)^{2}}-\alpha_{1} S+\alpha_{4} D+\alpha_{5}+\mu\right] z_{3} \\
& \leq\left[\begin{array}{l}
\alpha_{1} S-\alpha_{4} D-\alpha_{5}-\mu-\left(\alpha_{1}+\alpha_{4}\right) M \\
-\frac{\alpha_{2} S}{1+\alpha_{3} D}-\alpha_{6}-\mu_{D}
\end{array}\right]\left|z_{1}\right| \\
& +\left[-\alpha_{1} M-\alpha_{4} M\right]\left|z_{2}\right| \\
& +\left[\begin{array}{l}
\frac{\alpha_{2} S\left(1+\alpha_{3} D\right)}{\left(D \alpha_{3}+1\right)^{2}}-\frac{\alpha_{7} \alpha_{6}}{\alpha_{7}+\mu}-\alpha_{1} S+\alpha_{4} D \\
+\alpha_{5}+\mu
\end{array}\right]\left|z_{3}\right|
\end{aligned}
$$

Since $\left|z_{1}\right|+\left|z_{3}\right|>\left|z_{2}\right|$, we get

$$
\begin{aligned}
\leq & {\left[\begin{array}{l}
\alpha_{1} S-\alpha_{4} D-\alpha_{5}-\mu-\left(\alpha_{1}+\alpha_{4}\right) M-\frac{\alpha_{2} S}{1+\alpha_{3} D} \\
-\alpha_{6}-\mu_{D}
\end{array}\right]\left|z_{1}\right| } \\
& +\left[\begin{array}{l}
\frac{\alpha_{2} S\left(1+\alpha_{3} D\right)}{\left(D \alpha_{3}+1\right)^{2}}-\frac{\alpha_{7} \alpha_{6}}{\alpha_{7}+\mu}-\alpha_{1}(S+M) \\
-\alpha_{4}(M-D)+\alpha_{5}+\mu
\end{array}\right]\left|z_{3}\right|
\end{aligned}
$$

$D_{+}\|z\| \leq \max \left\{\alpha_{1} S-\alpha_{4} D-\alpha_{5}-\mu-\left(\alpha_{1}+\alpha_{4}\right) M\right.$

$$
\begin{aligned}
& -\frac{\alpha_{2} S}{1+\alpha_{3} D}-\alpha_{6}-\mu_{D}, \frac{\alpha_{2} S\left(1+\alpha_{3} D\right)}{\left(D \alpha_{3}+1\right)^{2}}-\frac{\alpha_{7} \alpha_{6}}{\alpha_{7}+\mu} \\
& \left.-\alpha_{1}(S+M)-\alpha_{4}(M-D)+\alpha_{5}+\mu\right\}\|z\|
\end{aligned}
$$

Case 8 If $z_{3}<0<z_{1}, z_{2}$ and $\left|z_{1}\right|+\left|z_{3}\right|<\left|z_{2}\right|$

then $\|z\|=\left|z_{2}\right|$ and

$D_{+}\|z\|=z_{2}^{\prime}=\left[\alpha_{4}\right] z_{1}$

$$
\begin{aligned}
& +\left[-\alpha_{1} M-\frac{\alpha_{2} \alpha_{3} S D}{1+\alpha_{3} D}-\frac{\alpha_{2} D}{D \alpha_{3}+1}-\mu\right] z_{2} \\
& +\left[\frac{\alpha_{7} \alpha_{5}}{\alpha_{7}+\mu}-\alpha_{1} S\right] z_{3}
\end{aligned}
$$

Since $\left|z_{1}\right|+\left|z_{3}\right|<\left|z_{2}\right|$, we get

$$
D_{+}\|z\| \leq\left[\begin{array}{l}
\frac{\alpha_{2} \alpha_{3} S D}{1+\alpha_{3} D}-\frac{\alpha_{2} D}{D \alpha_{3}+1}+\frac{\alpha_{7} \alpha_{5}}{\alpha_{7}+\mu} \\
-\alpha_{1}(M+S)+\alpha_{4}-\mu
\end{array}\right]\|z\|
$$

Combining all the eight cases, we got four independent inequality which are used in following theorem that proves the global stability of endemic eqilibrium point. Theorem 8. For $R_{0}>1$, the endemic equilibrium point $E^{*}$ is globally asymptotically stable if the following inequality holds

$$
\max \left(\chi_{1}, \chi_{2}, \chi_{3}, \chi_{4}\right)<-\chi
$$

Where,

$$
\begin{aligned}
& \chi_{1}=\max \left\{\begin{array}{l}
\alpha_{4}-\frac{\alpha_{2} D}{D \alpha_{3}+1}-\frac{\alpha_{2} \alpha_{3} S D}{1+\alpha_{3} D}-\mu, \frac{\alpha_{7} \alpha_{5}}{\alpha_{7}+\mu} \\
-\frac{\alpha_{2} \alpha_{3} S D}{\left(D \alpha_{3}+1\right)^{2}}-\alpha_{4} D-\alpha_{5}-\mu
\end{array}\right\} \\
& \chi_{2}=\max \left\{\alpha_{1} S-\alpha_{4} D-\alpha_{5}-\mu-\alpha_{4} M-\frac{\alpha_{2}(S+D)}{1+\alpha_{3} D}\right. \\
& -\alpha_{6}-\mu_{D}, \frac{\alpha_{2} S\left(1-\alpha_{3} D\right)}{\left(D \alpha_{3}+1\right)^{2}}-\frac{\alpha_{7} \alpha_{6}}{\alpha_{7}+\mu}+\alpha_{1}(S+M) \\
& \left.-\alpha_{4}(D+M)-\alpha_{5}-\mu\right\} \\
& \chi_{3}=\frac{\alpha_{2} D}{D \alpha_{3}+1}+\frac{\alpha_{2} \alpha_{3} S D}{1+\alpha_{3} D}-\frac{\alpha_{7} \alpha_{5}}{\alpha_{7}+\mu}+\alpha_{1}(M+S)-\alpha_{4}+\mu \\
& \chi_{4}=\frac{\alpha_{2} \alpha_{3} S D}{1+\alpha_{3} D}-\frac{\alpha_{2} D}{D \alpha_{3}+1}+\frac{\alpha_{7} \alpha_{5}}{\alpha_{7}+\mu}-\alpha_{1}(M+S)+\alpha_{4}-\mu
\end{aligned}
$$




\section{Optimal control}

Mosquitoes are the most prolific killers of humans in the animal kingdom. One of the most ancient and deadly diseases that mosquitoes transmit are malaria and dengue. It has been hypothesized due to influences on immune responses that infection with malaria can alter to the course of infection of the dengue. An effective way to protect the people from dengue who are already affected by malaria is to control vector. Also medication pays a major role to control spread of vector borne diseases.

In present dynamical model, two bounded Lebesgue integrable controls are introduced say $u_{1}$ and $u_{2} \cdot u_{1}$ control is to minimize concurrent infection cases by vector control and $u_{2}$ is a treatment control which helps to improve recovery rate. After applying control system (1) will take form as follow:

$$
\begin{aligned}
& \frac{d S}{d t}=B+\alpha_{7} R-\alpha_{1} S M-\frac{\alpha_{2} S D}{1+\alpha_{3} D}-\mu S \\
& \frac{d M}{d t}=\alpha_{1} S M-\alpha_{4} M D-u_{1} M-\alpha_{5} M-\mu M \\
& \frac{d D}{d t}=\frac{\alpha_{2} S D}{1+\alpha_{3} D}+\alpha_{4} M D+u_{1} M-\left(\alpha_{6}+u_{2}\right) D-\left(\mu+\mu_{D}\right) D \\
& \frac{d R}{d t}=\left(\alpha_{6}+u_{2}\right) D+\alpha_{5} M-\alpha_{7} R-\mu R
\end{aligned}
$$

The objective function $J\left(u_{i}, \Omega\right)$ for the mathematical model along with the optimal control is given by:

$$
J=\int_{0}^{T}\left(A_{1} S^{2}+A_{2} M^{2}+A_{3} D^{2}+A_{4} R^{2}+w_{1} u_{1}^{2}+w_{2} u_{2}^{2}\right) d t
$$

Here, $\Omega$ denotes set of all compartmental variables. $A_{i}$ are small positive constants to keep a balance in the size of the respective compartments. $w_{1}$ and $w_{2}$ are positive weight parameter which is associated with the control $u_{1}$ and $u_{2}$. The objective of our work is to maximize the total number of recovered individual by optimizing control variables $u_{1}$ and $u_{2}$.

As, the weight parameters $w_{1}$ and $w_{2}$ are constant of the control rates applied as vector control and treatment control, from which the optimal control condition is normalized. Now, we will calculate the values of control variables from $t=0$ to $t=T$ such that

$$
J\left(u_{1}(t), u_{2}(t)\right)=\text { optimum }\left\{J\left(u_{i}, \Omega\right) /\left(u_{1}, u_{2}\right) \in \phi\right\}
$$

Where $\phi$ is a smooth function on the interval $[0,1]$. The optimal controls denoted by $u_{1}^{*}$ and $u_{2}^{*}$ are founded by accumulating all the integrands of equation (4) using the lower bounds and upper bounds respectively with the results of Fleming and Rishel [11].
To optimize controls using the Pontryagin's principle we construct a Lagrangian function consisting of state equations and adjoint variables $\lambda_{1}, \lambda_{2}, \lambda_{3}, \lambda_{4}$ as follows:

$$
\begin{aligned}
L(\Omega, A)= & A_{1} S^{2}+A_{2} M^{2}+A_{3} D^{2}+A_{4} R^{2}+w_{1} u_{1}^{2}+w_{2} u_{2}^{2} \\
& +\lambda_{1}\left(B+\alpha_{7} R-\alpha_{1} S M-\frac{\alpha_{2} S D}{1+\alpha_{3} D}-\mu S\right) \\
& +\lambda_{2}\left(\alpha_{1} S M-\alpha_{4} M D-u_{1} M-\alpha_{5} M-\mu M\right) \\
& +\lambda_{3}\left(\frac{\alpha_{2} S D}{1+\alpha_{3} D}+\alpha_{4} M D+u_{1} M-\left(\alpha_{6}+u_{2}\right) D\right. \\
& \left.-\left(\mu+\mu_{D}\right) D\right)+\lambda_{4}\left(\left(\alpha_{6}+u_{2}\right) D+\alpha_{5} M\right. \\
& \left.-\alpha_{7} R-\mu R\right)
\end{aligned}
$$

The partially differentiation of the Lagrangian function with respect to each compartmental variable gives the adjoint equation variables $A_{i}=\left(\lambda_{1}, \lambda_{2}, \lambda_{3}, \lambda_{4}\right)$ corresponding to the system:

$$
\begin{aligned}
& \dot{\lambda}_{1}=-\frac{\partial L}{\partial S}=-2 A_{1} S+\left(\lambda_{1}-\lambda_{2}\right) \alpha_{1} M \\
& +\left(\lambda_{1}-\lambda_{3}\right) \frac{\alpha_{2} D}{1+\alpha_{3} D}+\mu \lambda_{1} \\
& \dot{\lambda}_{2}=-\frac{\partial L}{\partial M} \\
& =-2 A_{2} M+\left(\lambda_{1}-\lambda_{2}\right) \alpha_{1} S+\left(\lambda_{2}-\lambda_{3}\right)\left(\alpha_{4} D+u_{1}\right) \\
& +\left(\lambda_{2}-\lambda_{4}\right) \alpha_{5}+\lambda_{2} \mu-\frac{\lambda_{3} \alpha_{1} S}{1+\alpha_{3} D} \\
& \dot{\lambda}_{3}=-\frac{\partial L}{\partial D}=-2 A_{3} D+\left(\lambda_{1}-\lambda_{3}\right) \frac{\alpha_{2} S}{\left(1+\alpha_{3} D\right)^{2}} \\
& +\left(\lambda_{2}-\lambda_{3}\right) \alpha_{4} M+\left(\lambda_{3}-\lambda_{4}\right)\left(\alpha_{6}+u_{2}\right) \\
& +\lambda_{3}\left(\mu+\mu_{D}\right) \\
& \dot{\lambda}_{4}=-\frac{\partial L}{\partial R}=-2 A_{4} R+\left(\lambda_{4}-\lambda_{1}\right) \alpha_{7}+\lambda_{4} \mu
\end{aligned}
$$

The necessary conditions for Lagrangian function $L$ to be optimal are, $\dot{u}_{1}=0$ and $\dot{u}_{2}=0$. Hence we get,

$u_{1}=\frac{1}{2 w_{1}}\left(\lambda_{2}-\lambda_{3}\right) M, u_{2}=\frac{1}{2 w_{2}}\left(\lambda_{3}-\lambda_{4}\right) D$

Formulated required optimal controls are:

$$
\begin{aligned}
& u_{1}^{*}=\max \left\{a_{1}, \min \left(b_{1}, \frac{\left(\lambda_{2}-\lambda_{3}\right) M}{2 w_{1}}\right)\right\} \\
& u_{2}^{*}=\max \left\{a_{2}, \min \left(b_{2}, \frac{\left(\lambda_{3}-\lambda_{4}\right) D}{2 w_{2}}\right)\right\}
\end{aligned}
$$

Thus, analytical results for optimized controls have been visualised in simulation part. 


\section{Numerical simulation}

Bifurcation analysis helps to demonstrate the qualitative information about the equilibrium point. Figure 2 shows backward bifurcation diagram where $R_{C}=0.12$ is a critical point from which system's stability switches from unstable to stable state. If $R_{C}<R_{0}$, then for the point of $R_{0}$ backward bifurcation exists, moreover equilibrium coexist when $R_{C}<R_{0}<1$ [14], [29].

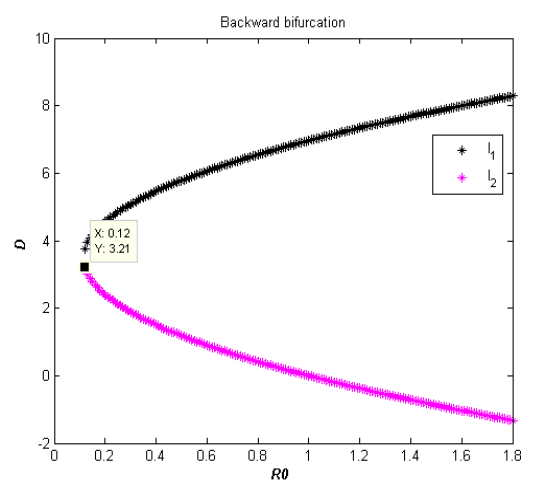

Figure 2. Bifurcation diagram for dengue infected individuals with $R_{0}$

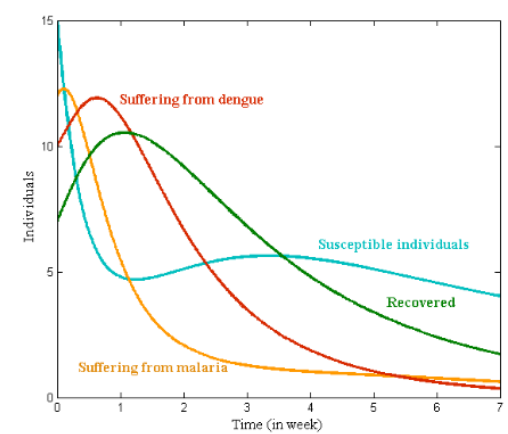

Figure 3. Time series of solution of malaria-dengue model

Figure 3 shows the flow of malaria-dengue model with time. It is observed that human immunity is more sensitive towards dengue compare to malaria infection moreover compare to dengue, recovery rate of malaria is higher. Hence we can say that medication is more effective on malaria infected compare to dengue infected. Compare to dengue, spread of malaria is easy to control by improving medication. Under proper medication both the diseases can be controlled in 7-8 weeks.

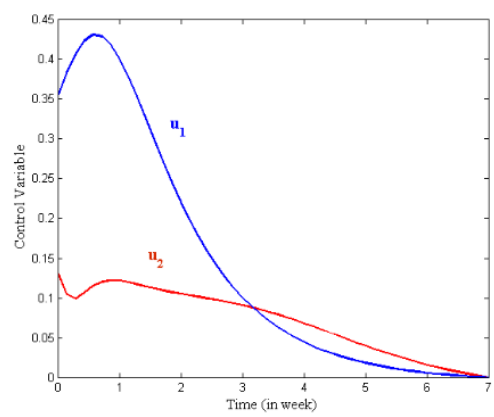

Figure 4. Variation in control variables with time
Figure 4 shows change in both the control variables needs to be done to stabilize the model. It is observed that initially $35 \%$ and $13 \%, u_{1}$ and $u_{2}$ controls are needed to be applied respectively.

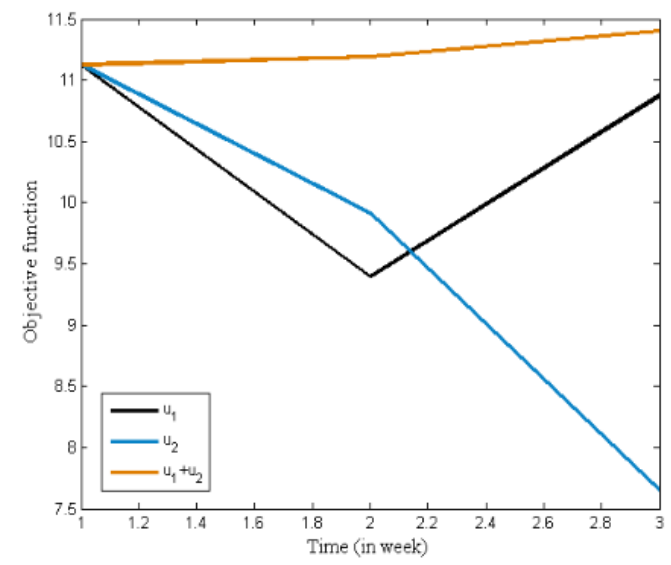

Figure 5. Change in objective function with time

Figure 5 gives change in objective function under influence of both the controls combine and individually which gives combine and individual effect of both the controls on malaria-dengue model. It is clearly visible that combine effect of controls gives more fruitful effect on the model compare to an individual effect.

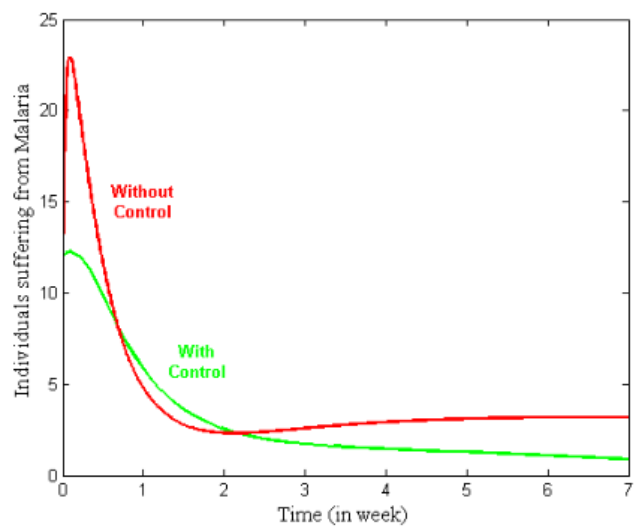

Figure 6. Impact of $u_{1}$ control on class of malaria infected individuals

The simulation in figure 6 interprets that that chances to get infected by malaria decreases by $50 \%$ after applying control $u_{1}$.

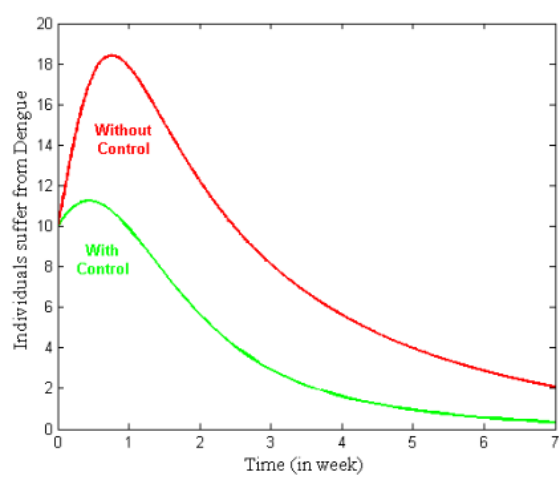

(a) Impact of $u_{1}$ control 


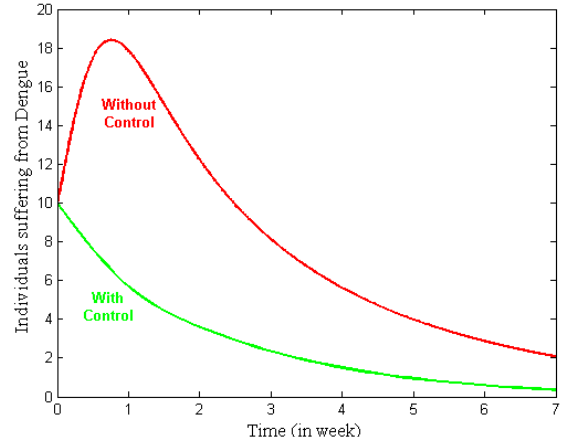

(b) Impact of $u_{2}$ control

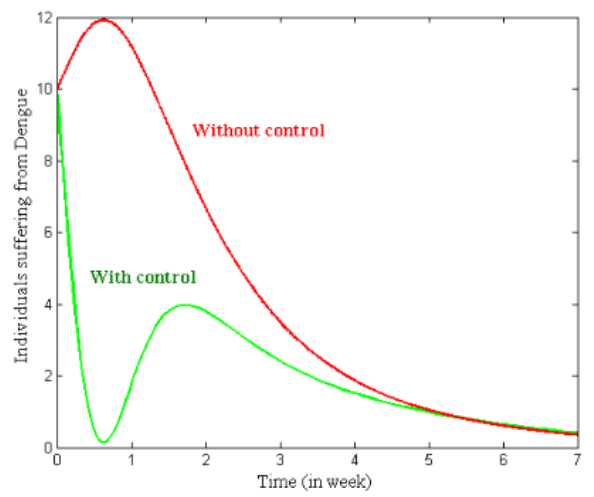

(c) Impact of both the controls

Figure 7. Impact of controls on class of dengue infected individuals

From figure 7(a) and 7(b), it is clear that for class of dengue infected individuals $u_{2}$ control is more effective compare to $u_{1}$ control moreover it is visualised in figure 7(c) that combine effect of both the control is even more effective which shoews only medication is not enough to minimize dengue infaction case, different acts which minimize concurrent infaction case also have a significant effect.

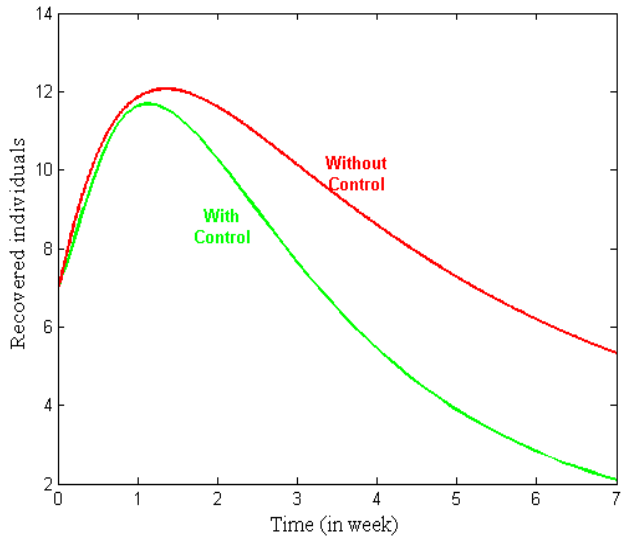

(b) Impact of $u_{1}$ control

Figure 8 shows separate and combine effect of both the controls on class of recovered individuals. From figure 8(c) we can observe better improvement in recovered class after applying both the controls at the same time. About $61 \%$ improvement is observed in recovery rate white applying both the control together.

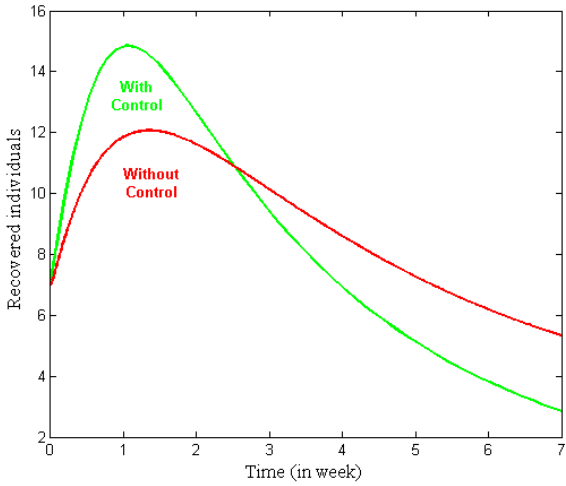

(b) Impact of $u_{2}$ control

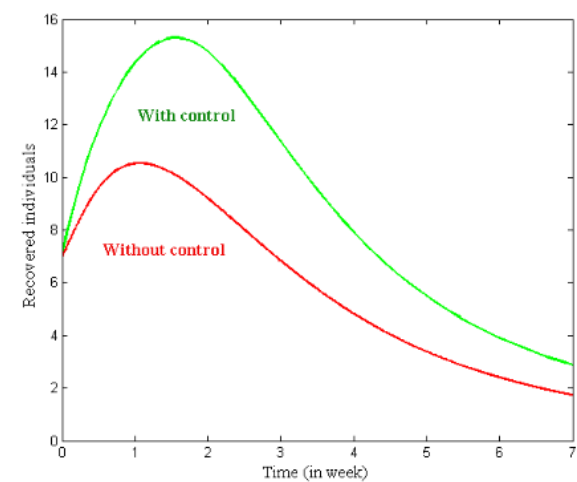

(c) Impact of both the controls

Figure 8. Impact of controls on class of recovered individuals

Also figure 8(a) and 8(b) deplicate that compare to $u_{1}$ control, $u_{2}$ control gives better result which suggest that madication plays a major contribution to control the concurrent infection. Hence better medication facility and avaibility is good approach to control outbreak of malaria-dengue infections in endemic areas.

\section{Conclusion}

The fight against most deadly mosquito-borne diseases malaria and dengue is a challenge to the world. In the present study, the system of dynamical model for two different mosquito borne diseases is studied through the use of mathematical modeling. Moreover, Optimal control theory is also applied on the model to visualise the effect of controles on it. The model have four equilibrium points for four different possible cases including disease free society, case when only one individual infection is present and the case when both the diseases are present concurrently in society. It is proved that all four equilibrium points are local and globally asymptotically stable under some parametric conditions. The formula of basic reproduction number $\left(R_{0}\right)$ used to calculate threshold value of the model. In this article, the basic reproduction number is formulated for malaria and dengue combinely, hence it 
is unaffected by parameter $\alpha_{4}$. Threshold value increases as value of parameters $\alpha_{1}$ and $\alpha_{2}$ is increases, and it decreases as $\alpha_{5}$ and $\alpha_{6}$ increases. Which simply means threshold value can be controled by improving recovery rates of both the diseases. Bifurcation analysis indicates that minimum rate of diseases spread is $12 \%$. Threshold value signifies that there is $14.9 \%$ chance to get infected by malaria and dengue concurrently. In numerical simulation we have observed the effect of optimal controls individually as well as concurrently and more stability is observed when we apply both the controls at same time. Also it is analysed that $61 \%$ improvement in recovery rate is observed under the effect of both optimal controls, which suggest that vector control by using insecticide, treated mosquito nets and indoor residual spraying and medication to improve recovery are the main way to prevent and reduce malaria and dengue transmission.

\section{Acknowledgments}

Second author (AHS) is funded by a Junior Research Fellowship from the Council of Scientific \& Industrial Research (CSIR) and all the authors are thankful to DST-FIST file \# MSI-097 for technical support to the Department of Mathematics.

\section{References}

[1] Aldila, D., \& Agustin, M. R. (2018, March). A Mathematical Model Of Dengue-Chikungunya CoInfection In A Closed Population. In Journal of Physics: Conference Series (Vol. 974, No. 1, p. 012001). IOP Publishing.

[2] Arino, J., McCluskey, C. C., \& van den Driessche, P. (2003). Global results for an epidemic model with vaccination that exhibits backward bifurcation. SIAM Journal on Applied Mathematics, 64(1), 260-276.

[3] Brauer, F., van den Driessche, P., \& Wu, J. (2008). Mathematical Epidemiology.lecture notes in mathematics-springer verlag-, 1(1945), 1-2.

[4] Carme, B., Matheus, S., Donutil, G., Raulin, O., Nacher, M., \& Morvan, J. (2009). Concurrent dengue and malaria in Cayenne hospital, French Guiana. Emerging infectious diseases, 15(4), 668671.

[5] Castillo-Chavez, C., Blower, S., van den Driessche, P., Kirschner, D., \& Yakubu, A. A. (Eds.). (2002). Mathematical approaches for emerging and reemerging infectious diseases: an introduction (Vol. 1). Springer Science \& Business Media.

[6] Charrel, R. N., Brouqui, P., Foucault, C., \& De Lamballerie, X. (2005). Concurrent dengue and malaria. Emerging infectious diseases, 11(7), 11531154

[7] Cox, J., Grillet, M. E., Ramos, O. M., Amador, M., \& Barrera, R. (2007). Habitat segregation of dengue vectors along an urban environmental gradient. The
American journal of tropical medicine and hygiene, 76(5), 820-826.

[8] Deresinski, S. (2006). Concurrent Plasmodium vivax malaria and dengue.

[9] Diekmann, O., Heesterbeek, J. A. P., \& Metz, J. A. (1990). On the definition and the computation of the basic reproduction ratio $\mathrm{R} 0$ in models for infectious diseases in heterogeneous populations. Journal of mathematical biology, 28(4), 365-382.

[10] Epelboin, L., Hanf, M., Dussart, P., Ouar-Epelboin, S., Djossou, F., Nacher, M., \& Carme, B. (2012). Is dengue and malaria co-infection more severe than single infections? A retrospective matched-pair study in French Guiana. Malaria journal, 11(1), 142-149.

[11] Fleming, W. H., Rishel, R. W., Marchuk, G. I., Balakrishnan, A. V., Borovkov, A. A., Makarov, V. L., Rubinov, A.M., Liptser, R.S., Shiryayev, A.N., Krassovsky, N.N. \& Subbotin, A. N. (1975). Applications of Mathematics. Deterministic and Stochastic Optimal Control.

[12] Isea, R., \& Lonngren, K. E. (2016). A preliminary mathematical model for the dynamic transmission of Dengue, Chikungunya and Zika. American Journal of Modern Physics and Application 2016; 3(2), 11 15 .

[13] Kaushik, R. M., Varma, A., Kaushik, R., \& Gaur, K. J. (2007). Concurrent dengue and malaria due to Plasmodium falciparum and P. vivax. Transactions of the Royal Society of Tropical Medicine and Hygiene, 101(10), 1048-1050.

[14] Khan, M. A., Islam, S., \& Khan, S. A. (2014). Mathematical modeling towards the dynamical interaction of leptospirosis. Applied Mathematics \& Information Sciences, 8(3), 1049-1056.

[15] Kumari, N., \& Sharma, S. (2018). Modeling the Dynamics of Infectious Disease Under the Influence of Environmental Pollution. International Journal of Applied and Computational Mathematics, 4, 1-24.

[16] Li, M. Y., \& Muldowney, J. S. (1996). A geometric approach to global-stability problems. SIAM Journal on Mathematical Analysis, 27(4), 1070-1083.

[17] Mackey, T. K., \& Liang, B. A. (2012). Lessons from SARS and H1N1/A: employing a WHO-WTO forum to promote optimal economic-public health pandemic response. Journal of public health policy, 33(1), 119-130.

[18] Mahmoud, D. M., Hussein, H. M., El Gozamy, B. M. R., Thabet, H. S., Hassan, M. A., \& Meselhey, R. A. A. (2019). Screening of Plasmodium parasite in vectors and humans in three villages in Aswan Governorate, Egypt. Journal of Parasitic Diseases, 43(1), 158-163.

[19] McCluskey, C. C., \& van den Driessche, P. (2004). Global analysis of two tuberculosis models. Journal of Dynamics and Differential Equations, 16(1), 139166. 
[20] Obsomer, V., Defourny, P., \& Coosemans, M. (2007). The Anopheles dirus complex: spatial distribution and environmental drivers. Malaria Journal, 6(1), 26-40.

[21] Patel, S. K., Rajora, N., Kumar, S., Sahu, A., Kochar, S. K., Krishna, C. M., \& Srivastava, S. (2019). Rapid discrimination of Malaria and Dengue Infected Patients Sera using Raman Spectroscopy. Analytical chemistry.

[22] Rodenhuis-Zybert, I. A., Wilschut, J., \& Smit, J. M. (2010). Dengue virus life cycle: viral and host factors modulating infectivity. Cellular and molecular life sciences, 67(16), 2773-2786.

[23] Rupali, P. (2019). Introduction to Tropical Medicine. Infectious Disease Clinics, 33(1), 1-15.

[24] Santana, V. D. S., Lavezzo, L. C., Mondini, A., Terzian, A. C. B., Bronzoni, R. V. D. M., Rossit, A. R. B., Machado, R.L.D., Rahal, P., Nogueira, M.C.L. \& Nogueira, M. L. (2010). Concurrent dengue and malaria in the Amazon region. Revista da Sociedade Brasileira de Medicina Tropical, 43(5), 508-511.

[25] Sharomi, O., Podder, C., Gumel, A., \& Song, B. (2008). Mathematical analysis of the transmission dynamics of HIV/TB coinfection in the presence of treatment. Mathematical Biosciences and Engineering, 5(1), 145-174.

[26] Silva, C. J., \& Torres, D. F. (2015). A TB-HIV/AIDS coinfection model and optimal control treatment. Discrete and Continuous Dynamical Systems, 35(9), 4639-4663.

[27] Smith, R. A. (1986). Some applications of Hausdorff dimension inequalities for ordinary differential equations. Proceedings of the Royal Society of Edinburgh Section A: Mathematics, 104(3-4), 235259.

[28] Van den Driessche, P., \& Watmough, J. (2002). Reproduction numbers and sub-threshold endemic equilibria for compartmental models of disease transmission. Mathematical biosciences, 180(1-2),
29-48.

[29] Wangari, I. M., Davis, S., \& Stone, L. (2016). Backward bifurcation in epidemic models: Problems arising with aggregated bifurcation parameters. Applied Mathematical Modelling, 40(2), 1669-1675.

[30] Ward, D. I. (2006). A case of fatal Plasmodium falciparum malaria complicated by acute dengue fever in East Timor. The American journal of tropical medicine and hygiene, 75(1), 182-185.

[31] Wiwanitkit, V. (2011). Concurrent malaria and dengue infection: a brief summary and comment. Asian Pacific journal of tropical biomedicine, 1(4), 326-327.

Nita H. Shah is a Professor in the Department of Mathematics, Gujarat University, Ahmedabad. She has 25 years of research experience in inventory management, forecasting and information technology and information systems. She has published 375+ articles in international journals, including APJOR (Singapore), International Journal of Production Economics, OMEGA, CCERO (Belgium), ECPE (Romania), Measuring, Control \& Simulation (France), JIOS (India), IJOMS (India), Industrial Engineering (India), European Journal of Operational Research, IJIR, IJOR, IJMOR, IJBPSCM.

(D) http://orcid.org/0000-0003-1605-4778

Ankush H. Suthar is research scholar in Department of Mathematics, Gujarat University, India. He is working in dynamical systems and mathematical modeling of health problem related to infectious diseases. He has published 2-papers in JMCS and IJCER on transmission of deadly infectious diseases like Nipah-virus and swine-flu.

(D) http://orcid.org/0000-0001-7583-450X

Ekta N. Jayswal is research scholar in Department of Mathematics, Gujarat University, India. She is working in finite dimensional dynamical systems and its stability. Her research interest is applications of mathematical modeling to resolve different environmental issues.

D http://orcid.org/0000-0002-5888-2478

An International Journal of Optimization and Control: Theories \& Applications (http://ijocta.balikesir.edu.tr)

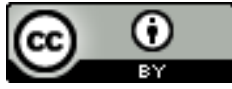

This work is licensed under a Creative Commons Attribution 4.0 International License. The authors retain ownership of the copyright for their article, but they allow anyone to download, reuse, reprint, modify, distribute, and/or copy articles in IJOCTA, so long as the original authors and source are credited. To see the complete license contents, please visit http://creativecommons.org/licenses/by/4.0/. 